\title{
MYC deregulates TET1 and TET2 expression to control global DNA (hydroxy)methylation and gene expression to maintain a neoplastic phenotype in T-ALL
}

\author{
Candace J. Poole ${ }^{1} \mathbb{B}$, Atul Lodh ${ }^{1}$, Jeong-Hyeon Choi $^{2}$ and Jan van Riggelen ${ }^{{ }^{*}}$
}

\begin{abstract}
Background: While aberrant DNA methylation is a characteristic feature of tumor cells, our knowledge of how these DNA methylation patterns are established and maintained is limited. DNA methyltransferases and ten-eleven translocation methylcytosine dioxygenases (TETs) function has been found altered in a variety of cancer types.

Results: Here, we report that in T cell acute lymphoblastic leukemia (T-ALL) the MYC oncogene controls the expression of TET1 and TET2 to maintain 5-methylcytosine $(5 \mathrm{mC})$ and 5 -hydroxymethylcytosine $(5 \mathrm{hmC})$ patterns, which is associated with tumor cell-specific gene expression. We found that cellular senescence and tumor regression upon MYC inactivation in T-ALL was associated with genome-wide changes in $5 \mathrm{mC}$ and $5 \mathrm{hmC}$ patterns. Correlating with the changes in DNA (hydroxy)methylation, we found that T-ALL overexpress TET1, while suppressing TET2 in a MYCdependent fashion. Consequently, MYC inactivation led to an inverse expression pattern, decreasing TET1, while increasing TET2 levels. Knockdown of TET1 or ectopic expression of TET2 in T-ALL was associated with genome-wide changes in $5 \mathrm{mC}$ and $5 \mathrm{hmC}$ enrichment and decreased cell proliferation, suggesting a tumor promoting function of TET1, and a tumor suppressing role for TET2. Among the genes and pathways controlled by TET1, we found ribosomal biogenesis and translational control of protein synthesis highly enriched.

Conclusions: Our finding that MYC directly deregulates the expression of TET1 and TET2 in T-ALL provides novel evidence that MYC controls DNA (hydroxy)methylation in a genome-wide fashion. It reveals a coordinated interplay between the components of the DNA (de)methylating machinery that contribute to MYC-driven tumor maintenance, highlighting the potential of specific TET enzymes for therapeutic strategies.
\end{abstract}

Keywords: MYC, TET1, TET2, DNA methylation, DNA hydroxymethylation, Leukemia/lymphoma

\section{Background}

The MYC oncogene is involved in the pathogenesis of $60-70 \%$ of all human cancers, including $\mathrm{T}$ cell acute lymphoblastic leukemia (T-ALL) (reviewed in $[1,2]$ ). MYC encodes for a transcription factor that controls the expression of a large number of genes, thereby deregulating a variety of cellular processes, ultimately leading to autonomous cell proliferation, growth, and angiogenesis,

\footnotetext{
*Correspondence: jvanriggelen@augusta.edu

1 Department of Biochemistry and Molecular Biology, Augusta University,

1410 Laney-Walker Blvd., Augusta, GA 30912, USA

Full list of author information is available at the end of the article
}

while blocking cellular differentiation (reviewed in [3, 4]). Its wide implications in human tumorigenesis and the notion that tumors can be dependent on enhanced MYC expression, exhibiting oncogene addiction, make the oncogene and its network a highly promising target for therapeutic strategies [5-7].

While the many functions of MYC were first attributed to its ability to both activate and repress transcription of a large number of genes through direct binding to their promoters, more and more reports challenge this dogma. In the classic model, MYC, heterodimerized with MAX, recognizes a DNA-motif named E-box (CACGTG 
), thereby transactivating canonical target genes through recruitment of chromatin-modifying cofactors $[8,9]$. At the same time, through interactions with other transcription factors such as MIZ-1, the transactivating function of MYC-MAX can be fine-tuned, or even inverted to repress the transcription of genes harboring an INR DNA-motif (reviewed in [10]). Furthermore, MYC has been demonstrated to bind virtually all active promoters and many enhancers, thereby boosting the transcriptional output of a given cell through various mechanisms [11-14]. In parallel, it has been emerging that MYC regulates chromatin structure in a genome-wide fashion [15-18], suggesting a mechanism that extends beyond the classic model of a site-specific transcription factor (reviewed in [19]).

We and others have demonstrated that cellular senescence accompanied by extensive chromatin remodeling is an important mechanism of tumor regression upon MYC inactivation in T-ALL, and other cancer types [16, 20, 21]. During this process, broad changes occur in histone methylation (increase of $\mathrm{H} 3 \mathrm{~K} 9 \mathrm{me} 3$ ) and acetylation (decrease of $\mathrm{H} 4 \mathrm{ac}$ ), suggesting that during tumor maintenance MYC maintains large areas of active chromatin. Consequently, MYC inactivation is associated with genome-wide changes in the epigenetic landscape. Indeed, there is growing evidence that MYC induces genome-wide alterations in chromatin in order to elicit its neoplastic properties (reviewed in [19]). As first evidence, N-MYC transcriptional upregulation of the histone acetyltransferase, GCN5, was reported to cause genome-wide acetylation of histones [15]. Similarly, we reported that MYC inactivation in T-ALL triggers genome-wide changes in histone acetylation and methylation associated with cellular senescence and tumor regression [16]. Furthermore, MYC recently has been shown to suppress chromatin regulators, SIN3B, HBP1, SUV420h1, and BTG via the miR-17-92 cluster [17]. Together, these results indicate that MYC controls genome-wide chromatin domains through modulating the expression of chromatin-modifying enzymes in order to create an epigenetic landscape that favors neoplastic gene expression programs.

Despite the recent reports teasing out the function of MYC as global regulator of transcription, it remains elusive how MYC establishes and maintains DNA methylation as an important component of chromatin structure. Tumor cells typically display global hypomethylation of repetitive DNA elements which contributes to genomic instability, while promoter and CpG island hypermethylation extinguish transcription of tumor suppressor genes. DNA methylation as 5-methylcytosine $(5 \mathrm{mC})$ is established by de novo DNA methyltransferases (DNMTs), DNMT3A and DNMT3B, while DNMT1 preferentially binds hemi-methylated DNA and maintains methylation to prevent passive demethylation (reviewed in [22]). Aberrant DNA methylation is a characteristic feature of tumor cells and is known to contribute to tumorigenesis in human neoplasia [23-25]. Shedding light on how MYC controls DNA methylation in T-ALL and Burkitt lymphoma, we recently reported that MYC causes the overexpression of $D N M T 3 B$, maintaining specific $5 \mathrm{mC}$ and thus gene expression patterns which are important for tumor maintenance [26]. However, the role of teneleven translocation methylcytosine dioxygenases (TETs) in this context remained elusive.

TET enzymes convert $5 \mathrm{mC}$ to 5 -hydroxymethylcytosine $(5 \mathrm{hmC})$ and other cytosine intermediates (5-formylcytosine $(5 \mathrm{fC})$ and 5-carboxycytosine $(5 \mathrm{caC})$ ), which contribute to the process of active DNA demethylation through base excision repair processes. Opposite to the repressive effects $5 \mathrm{mC}$ often has at promoters, the enrichment of $5 \mathrm{hmC}$ at gene promoters correlates with increased gene expression [27-30]. It has been reported that genome-wide distribution of $5 \mathrm{hmC}$ is overall reduced in neoplastic tissue and tumor cell-specific $5 \mathrm{hmC}$ occurs at specific gene coding regions, revealing the importance of $5 \mathrm{hmC}$ in modulating gene expression $[27,31]$. However, much remains elusive how $5 \mathrm{mC}$ and $5 \mathrm{hmC}$ patterns contribute to the deregulation of gene expression during MYC-driven tumorigenesis and tumor maintenance.

Understanding the molecular mechanisms how tumor cell-specific DNA (hydroxy)methylation patterns are established and maintained by MYC may provide novel therapeutic strategies, aiming at specific components of the DNA (de)methylating machinery. Here, we report that in T-ALL, the MYC oncogene controls the expression of both TET1 and TET2, which in turn contribute to tumor cell-specific $5 \mathrm{mC}$ and $5 \mathrm{hmC}$ patterns in a genomewide fashion with importance for tumor maintenance.

\section{Results}

Tumor regression upon MYC inactivation in T-ALL is associated with genome-wide changes in DNA (hydroxy) methylation patterns

Inactivation of the MYC oncogene in a mouse model of T-ALL (E $\mu S R \alpha-t T A \alpha$;tet-o-MYC) causes sustained tumor regression by eliciting the phenomenon of oncogene addiction [5]. Using this T-ALL model, we have previously demonstrated that tumor regression upon MYC inactivation depends on activation of cellular senescence pathways associated with genome-wide changes in chromatin structure including histone acetylation (decrease of $\mathrm{H} 4 \mathrm{ac}$ ) and methylation (increase of $\mathrm{H} 3 \mathrm{~K} 9 \mathrm{me} 3$ ), associated with heterochromatin formation $[16,21,32]$. Furthermore, we recently reported that 
MYC deregulates the expression of methylation modifiers, DNMT3B and DNMT1, essential for tumor maintenance [26]. Together, these findings suggest that during tumor maintenance MYC maintains large domains of active chromatin, and that tumor regression upon MYC inactivation is tightly linked to changes in expression of chromatin modifiers resulting in genome-wide changes to chromatin structure.

To determine the effect MYC inactivation in tumors has on DNA methylation $(5 \mathrm{mC})$ and hydroxymethylation $(5 \mathrm{hmC})$, we carried out methylated DNA immunoprecipitation (MeDIP- and hMeDIP-seq, respectively) analysis, taking advantage of the tetracycline-regulated $c$-myc allele in T-ALL cells derived from E $\mu S R \alpha$ tTA $\alpha$;tet-o-MYC mice (Fig. 1). We compared mouse T-ALL cells (6780) in vitro before (CTRL) and upon inactivation of MYC by adding $20 \mathrm{ng} / \mathrm{mL}$ doxycycline (+DOX) to the culture medium for 2 days. MYC inactivation was validated by RT-qPCR (Additional file 1: Fig. S1). For each sample, 45-60 million Illumina sequencing reads were generated. Of these, $\sim 45-80 \%$ were successfully mapped to either strand of the mouse genome (mm10). To identify significantly differentially methylated regions (DMRs) and differentially hydroxymethylated regions (hDMRs), we performed a genome-wide, unbiased DMR and hDMR detection using a complete tiling of the mouse genome using a cutoff of $\log 2 \mathrm{FC} \geq 1$ with a $P$ value of $\leq 10^{-4}$.

We identified a total of 615,875 DMRs and 545,504 hDMRs that become significantly hypo- or hypermethylated upon MYC inactivation for 2 days. The genomic location of DMRs and hDMRs between MYC on and off states, displayed as circular plot, indicates genome-wide changes in $5 \mathrm{mC}$ and $5 \mathrm{hmC}$ distribution (Fig. 1a). We next mapped the DMRs and hDMRs (both hypo- and hypermethylated) to the annotated RefSeq genes in the mouse genome. We found 366 and 323 DMRs and hDMRs associated with mRNAs, 186 and 123 with enhancers, 1113 and 1093 with super-enhancers, 613,839 and 543,625 with small noncoding RNAs (ncRNAs), and 358 and 326 with long ncRNAs, respectively (Fig. 1b). We further annotated DMRs and hDMRs associated with proteincoding genes for cis-regulatory elements. Of the those DMRs and hDMRs, we found $3.11 \%$ and $1.78 \%$ associated with CpG islands, $17.46 \%$ and $9.20 \%$ with exons, $40.67 \%$ and $52.23 \%$ introns, $4.07 \%$ and with $3.26 \% 5^{\prime}$ - or $3^{\prime}$-UTRs, and $34.69 \%$ and $33.53 \%$ with sequences $2 \mathrm{kbp}$ or more upstream or downstream of the nearest gene, respectively (Fig. 1c). The fold change of DMRs and hDMRs associated with protein coding genes is displayed as heatmap, respectively (Fig. 1d, e). Of the 366 and 323 genes associated with DMRs and hDMRs, we found 275 and
240 to increase, and 91 and 83 to decrease in (hydroxy) methylation, respectively.

To determine the biological processes associated with DNA methylation changes upon MYC inactivation in T-ALL, we performed gene ontology analysis using the Database for Annotation, Visualization and Integrated Discovery (DAVID). Consistent with MYC's broad reach, we found a wide variety of processes associated with DMRs and hDMRs, ranging from regulation of cell growth and proliferation, to differentiation and metabolism (Fig. If and Additional file 3). Taken together, the MeDIP- and hMeDIP-seq analyses reveal genome-wide changes in $5 \mathrm{mC}$ and $5 \mathrm{hmC}$ distribution associated with a wide variety of biological processes upon MYC inactivation, indicating that MYC maintains tumor cell-specific DNA (hydroxy)methylation patterns in T-ALL.

\section{TET1 and TET2 expression levels in T-ALL are} MYC-dependent and are inversed upon MYC inactivation We previously reported that MYC causes the overexpression of DNMT1 and DNMT3B in T-ALL, thereby establishing and maintaining specific $5 \mathrm{mC}$ and thus gene expression patterns [26]. To further investigate the mechanism underlying global $5 \mathrm{mC}$ and $5 \mathrm{hmC}$ changes upon MYC inactivation, we performed gene expression profiling for TET enzymes (Fig. 2). We compared T-ALL cells (6780) derived from $E \mu S R \alpha-t T A \alpha$;tet-o-MYC mice, harboring a tetracycline-regulated c-myc allele, before (CTRL) and upon MYC inactivation (+DOX) over the course of 3 days. RT-qPCR analysis for $M Y C$ and its canonical target gene, Ornithine Decarboxylase 1 (ODC1), verified MYC inactivation (96.17-fold, $P=8.41 \times 10^{-9}$, and 12.36-fold, $P=1.2 \times 10^{-5}$ lower on

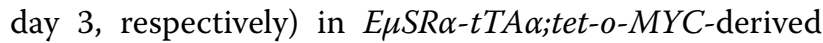
T-ALL cells (Fig. 2a). Subsequently, RT-qPCR for TET1 and TET2 revealed a direct correlation between $M Y C$ and TET1 expression levels, while showing an inverse correlation between $M Y C$ and TET2 levels (Fig. 2b). In mouse T-ALL, TET1 mRNA levels decreased significantly (6.63-fold, $P=0.021$ ), while TET2 mRNA levels increased significantly $\left(2.55\right.$-fold, $\left.P=9.4 \times 10^{-5}\right)$ upon MYC inactivation after 3 days.

Furthermore, to validate our results in mouse T-ALL we used P493-6 cells as model for high and low MYC expression in human lymphocytes, allowing for MYC inactivation. We compared human Burkitt lymphomalike cells (P493-6), harboring a tetracycline-regulated c-myc allele, before (CTL) and upon MYC inactivation (+DOX) over the course of 2 days. RT-qPCR showed a significant decrease $\left(64.39\right.$-fold, $\left.P=7.67 \times 10^{-6}\right)$ in $M Y C$ mRNA expression upon 2 days of DOX treatment (Fig. 2c). Mimicking our results in T-ALL, TET1 mRNA decreased (5.25-fold, $P=0.0015)$, while TET2 


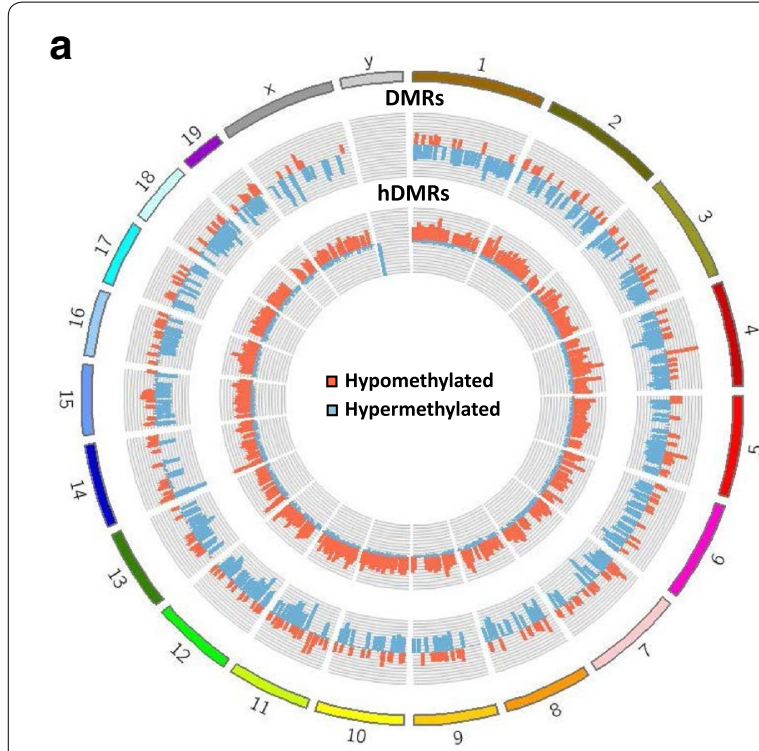

b
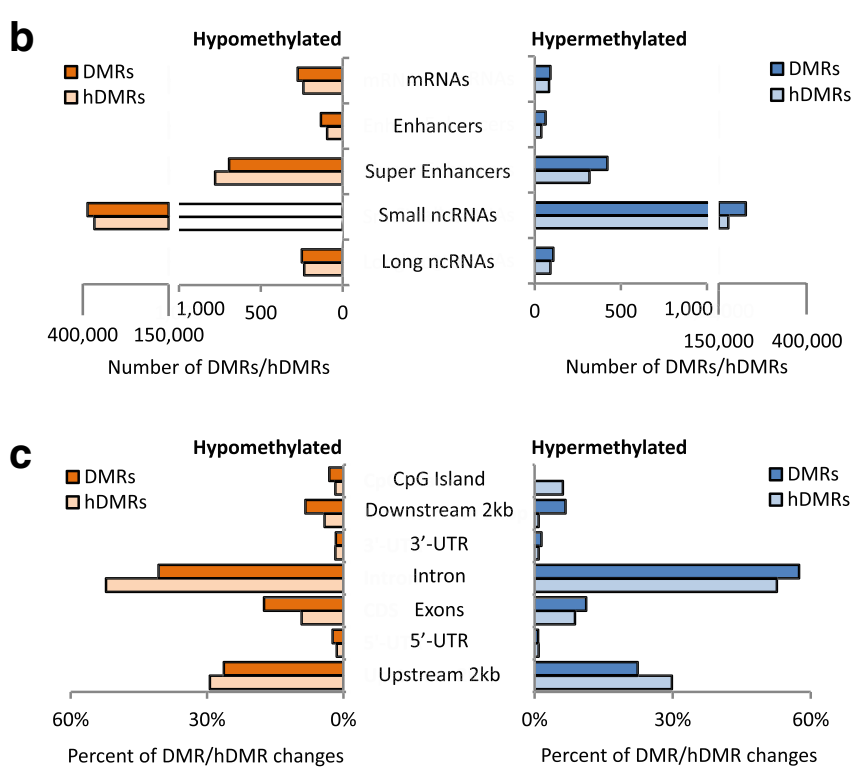

d

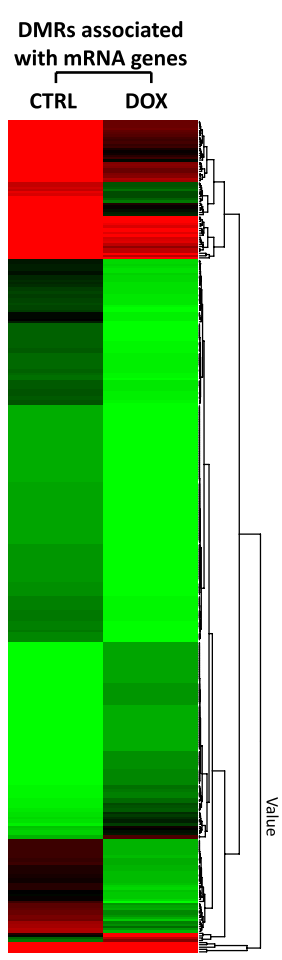

e

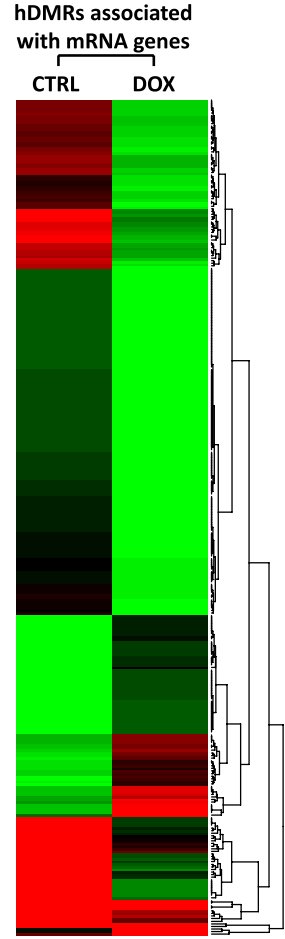

f

Biological Processes associated with DMRs and hDMRs

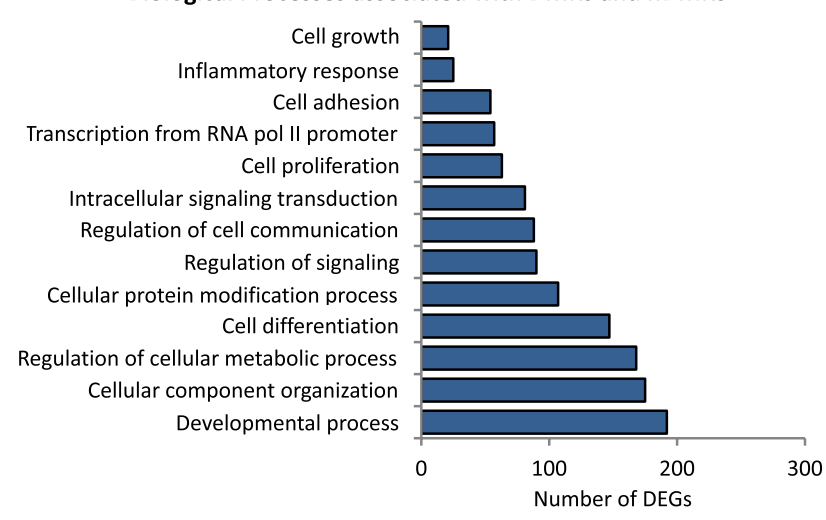

Fig. 1 Tumor regression upon MYC inactivation in T-ALL is associated with genome-wide changes in DNA (hydroxy)methylation. MeDIP- and hMeDIP-seq analysis of T-ALL cells (6780) derived from E $\mu$ SRa-tTAa;tet-o-MYC mice before and upon MYC inactivation through treatment with $20 \mathrm{ng} /$ $\mathrm{mL}$ DOX for 2 days. a Genomic distribution of DMRs and hDMRs is displayed as chromosome-based circular plot. Cutoff: log2FC $\geq 1$ with a $P$ value of $\leq 10^{-4}$. b Hypo- or hypermethylated DMRs and hDMRs are shown annotated for their association with mRNAs, enhancers, super-enhancers, small noncoding RNAs, and long noncoding RNAs. c Hypo- or hypermethylated DMRs and hDMRs associated with mRNAs are shown annotated for cis-regulatory elements: CpG islands, exons, introns, 5'-/3'-UTRs, and sequences 2 kbp upstream or downstream of the nearest gene. Heatmap showing hierarchical clustering of $\mathbf{d}$ DMRs and e hDMRs associated with protein-coding genes. Gene names are listed in Additional file 3. $\mathbf{f}$ Gene ontology analysis (DAVID) indicating biological processes associated with DMRs and hDMRs 
a

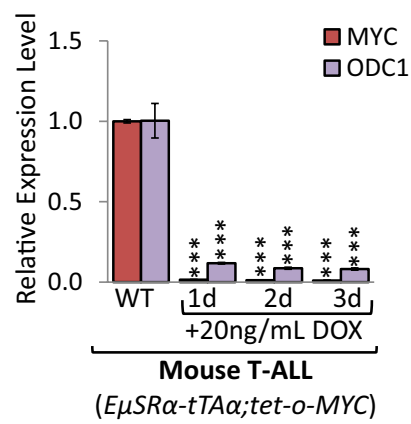

b

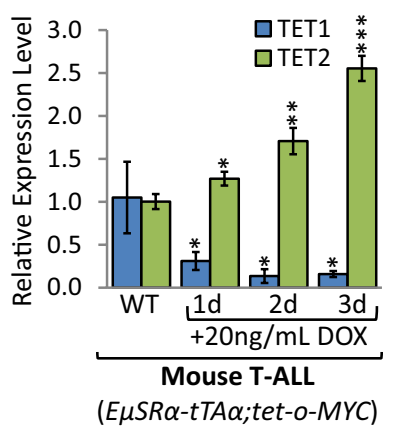

C

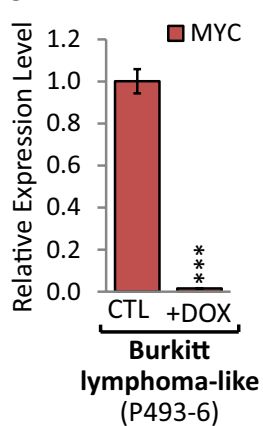

d

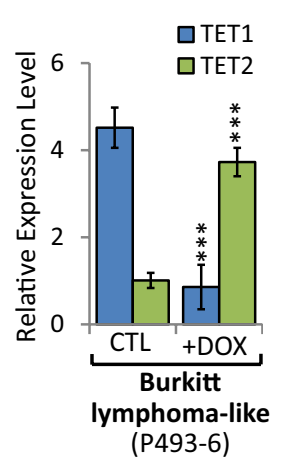

e

Burkitt lymphoma-like (P493-6)

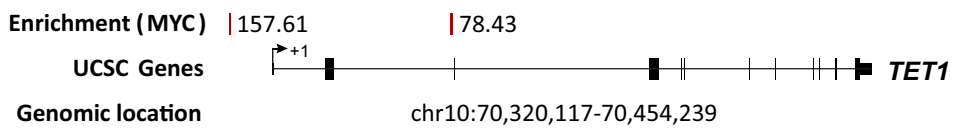

Enrichment (MYC) | 361.05

UCSC Genes

$\overrightarrow{+1}$

chr4:106,067,842-106,200,960

Fig. 2 TET1 and TET2 levels are dependent on MYC expression. MYC inactivation in T-ALL cells (6780) derived from EMSRa-tTAa;tet-o-MYC mice, and human Burkitt lymphoma-like (P493-6) cells, harboring a tetracycline-regulated C-MYC allele, in a time-dependent manner for 1, 2, and 3 days using $20 \mathrm{ng} / \mathrm{mL}$ DOX. Mouse T-ALL cells: a RT-qPCR analysis of MYC and its canonical target gene Ornithine Decarboxylase 1 (ODC1), and $\mathbf{b}$ of TET1 and TET2. RT-qPCR data were normalized to UBC. Human Burkitt lymphoma-like cells: c RT-qPCR of MYC and c TET1 and TET2 in P493-6 cells before (CTL) and upon MYC inactivation through treatment with $20 \mathrm{ng} / \mathrm{mL}$ DOX for 2 days (+DOX). RT-qPCR data were normalized to RPL13A. d MYC ChIP-seq data for P493-6 cells obtained from Sabo et al. [13] indicating enrichment score for MYC at the TET1 and TET2 loci. Traces were generated based on reference genome hg19 using the UCSC Genome Browser. The chromosomal location is indicated in bp. MYC binding peaks are displayed as red vertical bars; numbers represent the relative fold enrichment for MYC. Exons are displayed as black vertical bars, the UTR is represented by a black line, and the transcription start site (TSS) is marked by an arrow indicating the direction of transcription

levels increased (3.72-fold, $P=2.20 \times 10^{-5}$ ) (Fig. 2d). Together, these results indicate that TET1 expression is high, while TET2 is low in T-ALL derived from $E \mu S R \alpha$ -

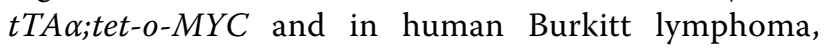
revealing a direct correlation between $M Y C$ and TET1, and an inverse correlation between $M Y C$ and TET2 expression levels.

To further determine whether MYC directly binds to the TET1 and TET2 genes and controls their transcription, we analyzed chromatin immunoprecipitation (ChIP-seq) data for P493-6 cells generated by Sabo et al. [13] (see Fig. 2e). We found MYC binding to the genomic TET1 and TET2 loci to be significantly enriched in P493-6 cells, revealing that MYC occupies sequences at the TET1 locus upstream of exon 1 (enrichment 157.61) and exon 3 (enrichment 78.43), as well as the TET2 locus downstream of exon 1 (enrichment 361.05). We conclude that high TET1 and low TET2 expression levels in mouse T-ALL and human Burkitt lymphoma-like cells are directly MYC-dependent and are inversed upon MYC inactivation.

\section{TET1 is overexpressed, while TET 2 is suppressed in human T-ALL cell lines and clinical specimens}

To translate our results from transgenic models to patient-derived cell lines overexpressing endogenous MYC, we performed gene expression profiling for TET1 and TET2, comparing a panel of human T-ALL cell lines to peripheral blood mononuclear cells (PBMCs) and spleen obtained from healthy donors (Fig. 3a-c). RT-qPCR analysis indicated that $M Y C$ mRNA levels were significantly higher in CCRF-CEM (177.43-fold), MOLT3 (7.04-fold), MOLT4 (32.93-fold), CCRF-HSB2 (3.89-fold), and JURKAT (8.08-fold), compared to normal PBMCs. RT-qPCR analysis indicated that TET1 mRNA levels were significantly higher in CCRF-CEM (63.80-fold), MOLT3 (13.23-fold), 

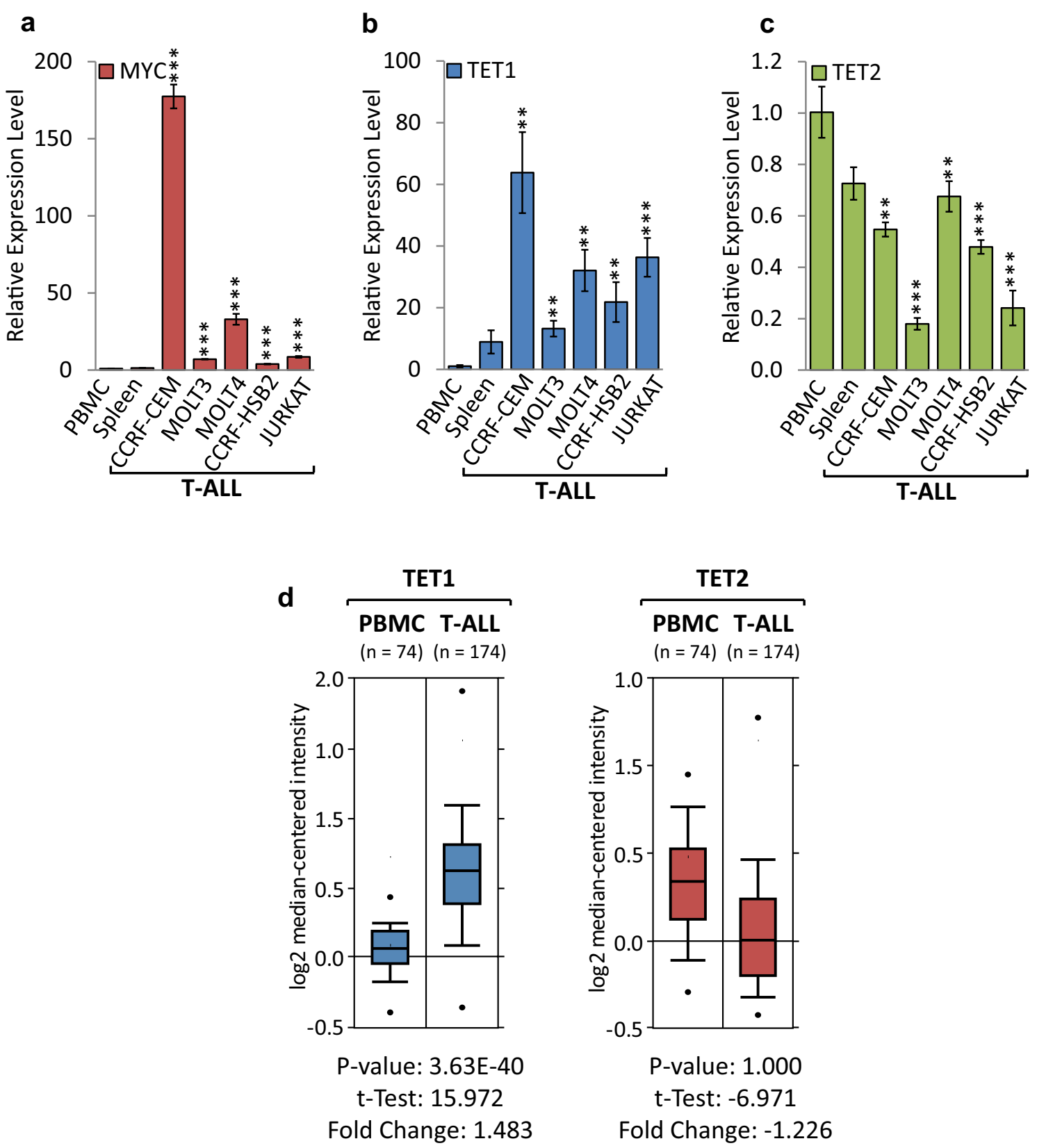

Fig. 3 TET1 is overexpressed, while TET2 is suppressed in human T-ALL cell lines and clinical specimens. Expression profiling of TET1 and TET2 in human T-ALL cell lines and clinical specimens compared to non-malignant tissue. RT-qPCR of a MYC, b TET1 and c TET2 in human T-ALL (CCRF-CEM, MOLT3, MOLT4, CCRF-HSB2, JURKAT) compared to peripheral blood mononuclear cells (PBMCs) and spleen obtained from healthy donors. RT-qPCR data were normalized to RPL13A. Error bars represent mean \pm SEM; $n=3$; two-tailed Student's $t$ test: ${ }^{*} P<0.05 ;{ }^{* *} P<0.01$; ${ }^{* * *} P<0.001$. d TET1 and TET2 expression profiles in clinical specimens obtained though Oncomine (http://www.oncomine.org) from Haferlach et al. [33]. TET1 and TET2 mRNA levels are displayed for T-ALL $(n=174)$ compared to PBMCs $(n=74)$. Boxes indicate the interquartile range; the line within the box represents the median. Whiskers indicate the non-outlier minimum and maximum. Outliers are represented by circles. Significant $P$ values and fold changes are indicated

MOLT4 (32.07-fold), CCRF-HSB2 (21.83-fold), and JURKAT (36.34-fold), compared to normal PBMCs. In contrast, we found TET2 expression to be significantly lower in CCRF-CEM (1.83-fold), MOLT3 (5.56-fold), MOLT4 (1.48-fold), CCRF-HSB2 (2.09-fold), and JURKAT (4.14-fold). To test whether clinical T-ALL specimens resemble our results from established cell 
lines, we analyzed publically available expression data obtained from Haferlach et al. [33] via Oncomine (Fig. 3d). We found that TET1 mRNA expression levels are elevated (1.483-fold, $\left.P=3.63 \times 10^{-40}\right)$, while TET2 expression levels are lower (1.226-fold, $P=1.0$ ) in clinical T-ALL $(n=174)$ than in normal PBMCs $(n=74)$, which is consistent with our findings in cell lines. Taken together, the expression profiling reveals that TET1 is overexpressed, while TET2 is suppressed in patient-derived T-ALL cell lines and clinical specimens, mimicking the above results from transgenic models.

\section{TET1 knockdown reduces cell proliferation of human T-ALL cells}

To determine whether loss of TET1 function affects tumor cell proliferation and viability, we carried out shRNA-mediated TET1 knockdown (KD) in human T-ALL cells (CCRF-CEM). CCRF-CEM is an established T-ALL cell line, exhibiting high MYC expression. We compared CCRF-CEM cells upon shRNA-mediated knockdown of TET1 to a shRNA control (Fig. 4). First, we validated TET1 KD as well as MYC, TET2, and TET3 expression levels by RT-qPCR (Fig. 4a, b). Compared to scrambled (SCR) control, CCRF-CEM cells expressing TET1sh show 3.63-fold less TET1 mRNA levels, while $M Y C$, TET2, and TET3 levels were similar. The corresponding growth curve indicates that TET1 KD in CCRFCEM decreased cell proliferation (Fig. 4c). To determine a mechanism underlying reduced cell proliferation, flow cytometric cell viability and cell cycle analysis were performed. Cell cycle analysis based on propidium iodide (PI) confirmed a decrease in CCRF-CEM cell proliferation, indicating a significant decrease in G1 phase cells from $45.2 \%$ for SCR to $40.3 \%$ for TET1sh, and an increase in $\mathrm{G} 2 / \mathrm{M}$ phase cells from $28.1 \%$ for SCR to $37.3 \%$ for TET1sh (Fig. 4d). In parallel, we quantified cell death by Annexin V and PI staining followed by flow cytometric analysis (Fig. 4e). While we did not detect significant changes in the fraction of necrotic cells, we found a small decrease in apoptotic cells for TET1sh. We furthermore validated our findings in mouse T-ALL cells, where TET1 KD using a different shRNA reduced tumor cell proliferation through cell cycle arrest (Additional file 2: Fig. S2). We conclude that loss of TET1 expression in both mouse and human T-ALL cells leads to reduced cell proliferation primarily through cell cycle arrest mechanisms.

\section{Knockdown of TET1 in human T-ALL cells alters DNA (hydroxy)methylation patterns and gene expression programs}

To investigate the molecular mechanisms underlying reduced tumor cell proliferation upon TET1 KD in human T-ALL cells, we measured DNA (hydroxy)methylation and RNA expression changes, using MeDIP-, hMeDIP-, and RNA-seq analysis, comparing CCRF-CEM cells before (SCR) and upon TET1 KD (TET1sh) (Fig. 5). For each sample, we generated 30-40 million Illumina sequencing reads, of which $\sim 40-85 \%$ were successfully mapped to either strand of the human genome (hg19). To identify statistically significant DMRs and hDMRs, we used a cutoff of $\log 2 \mathrm{FC} \geq 1$ with a $P$ value of $\leq 10^{-4}$.

We identified a total of 17,712 DMRs and 31,253 hDMRs that increased or decreased significantly upon TET1 KD in CCRF-CEM cells. The chromosomebased circular plot reveals genome-wide changes in the $5 \mathrm{mC}$ and $5 \mathrm{hmC}$ patterns upon loss of TET1 expression (Fig. 5a). Annotating DMRs and hDMRs (both hypo- and hypermethylated) with the nearest gene, we found 259 and 277 DMRs and hDMRs associated with mRNAs, 12,386 and 22,786 with enhancers, 2011 and 3068 with super-enhancers, 2770 and 5054 with small ncRNAs, and 295 and 378 with long ncRNAs, respectively (Fig. 5b). By further annotating DMRs and hDMRs associated with protein-coding genes with cis-regulatory elements, we found $3.23 \%$ and $2.76 \%$ of the DMRs and hDMRs associated with CpG islands, $91.58 \%$ and $93.40 \%$ with introns, $6.04 \%$ and $3.91 \%$ with exons, $2.05 \%$ and $0.93 \%$ with $5^{\prime}$ or $3^{\prime}$-UTRs, and $0 \%$ and $1.49 \%$ with sequences $2 \mathrm{kbp}$ upstream or downstream of the nearest gene, respectively (Fig. 5c). Thus, TET1 KD in human T-ALL cells leads to genome-wide changes in DNA (hydroxy)methylation patterns associated mostly with introns.

To determine the effect of alterations in DNA (hydroxy) methylation upon TET1 KD on RNA expression in CCRF-CEM cells, we next performed RNA-seq. We identified 3300 statistically significant DEGs (Fig. 5d). Of those, 1806 decreased, while 1494 increased in expression upon TET1 KD. To determine the biological processes associated with the DEGs, we performed gene ontology analysis using DAVID. We found a wide variety of processes including regulation of transcription, RNA metabolism, intracellular signaling as well as cell cycle and cell death-related pathways to be affected (Fig. 5e and Additional file 3). To further associate RNA expression with biological processes, we performed gene set enrichment analysis (GSEA). Among the largest enrichments, we found ribosome biogenesis $(n=272)$, ribosome assembly $(n=60)$, translational initiation $(n=136)$, and tRNA processing $(n=81)$, all associated with protein synthesis (Fig. 5f). Together, these results indicate that reduced proliferation of T-ALL cells upon loss of TET1 function is associated with decreased global protein synthesis. Similarly, inactivation of MYC in T-ALL has previously been shown to shut down global protein synthesis 

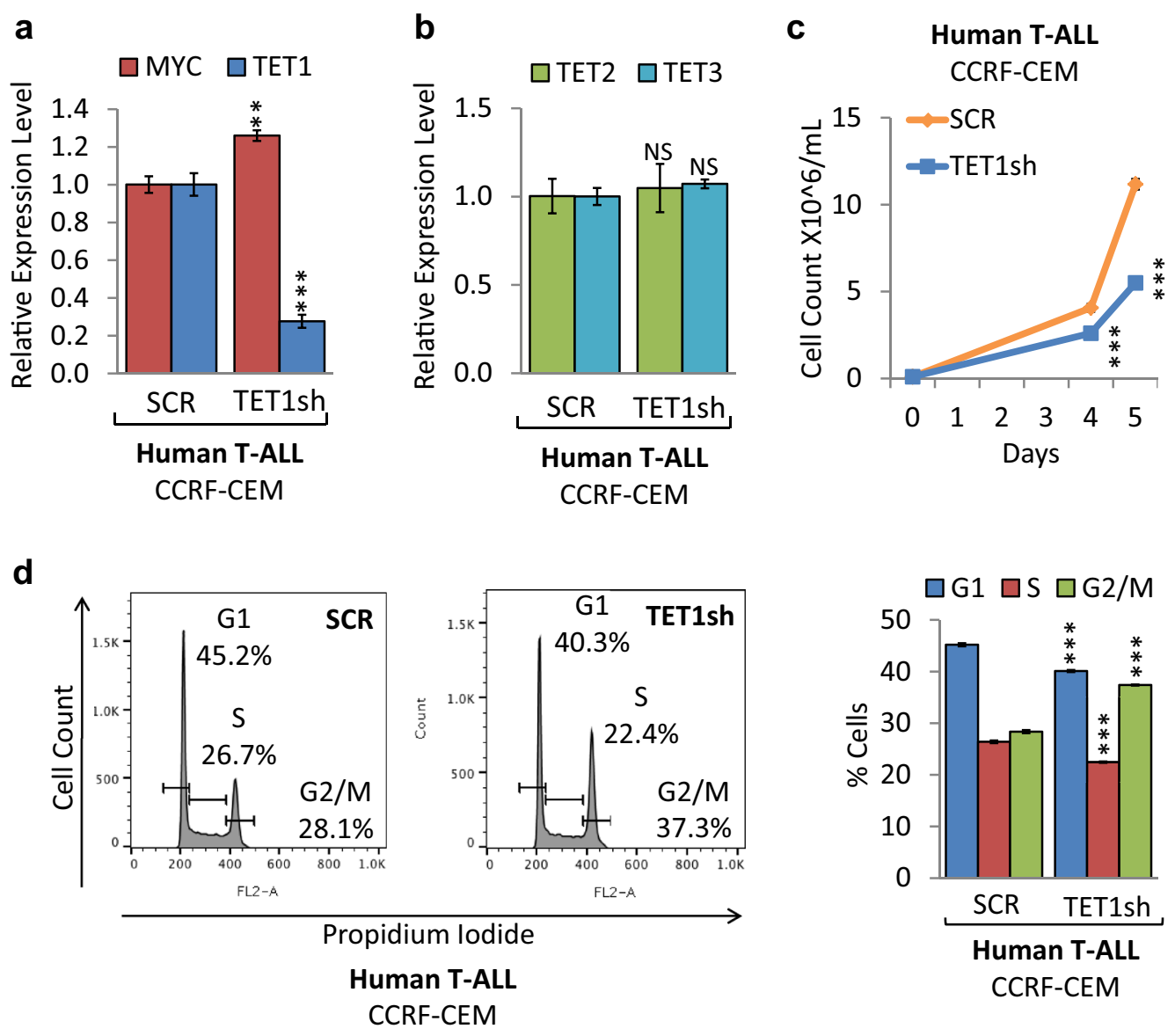

e
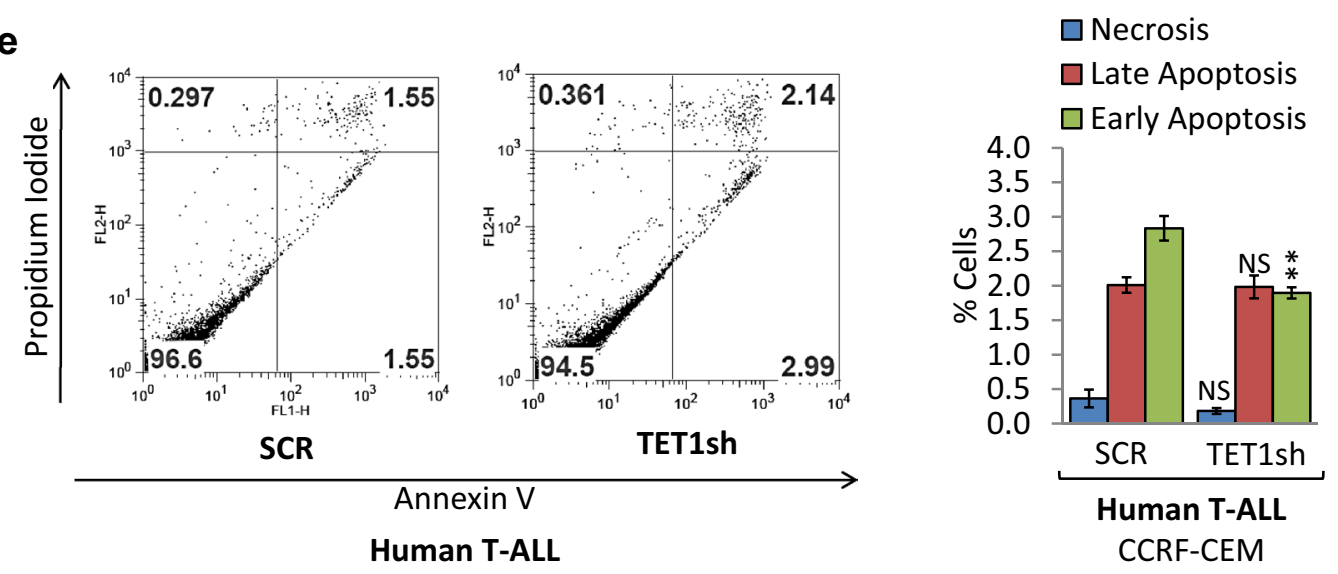

Human T-ALL

CCRF-CEM

Fig. 4 TET1 KD reduces cell proliferation of human T-ALL. Human T-ALL cells (CCRF-CEM) were compared before (SCR) and upon TET1 KD (TET1sh). RT-qPCR of a MYC and TET1, b TET2 and TET3. RT-qPCR data were normalized to RPL13a. c Growth curve comparing viable cell counts. $\mathbf{d}$ Flow cytometric cell cycle analysis based on propidium iodide (PI) staining. The cell cycle distribution (G1, S, and G2/M) is displayed in percent. e Flow cytometric analysis of apoptosis based on Annexin V/PI staining. Flow cytometry profile of Annexin V staining ( $X$ axis) and PI ( $Y$ axis) is shown for representative samples. The lower right quadrant indicates the percentage of early apoptotic cells in each condition; the upper right quadrant indicates the percentage of late apoptotic cells; the upper left quadrant indicates the percentage of necrotic cells. Apoptotic cells (Annexin $V$-positive cells) are displayed as the percentage of gated cells. Error bars represent mean $\pm S E M ; n=3$; two-tailed Student's $t$ test: ${ }^{*} P<0.05$; ${ }^{* *} P<0.01 ;{ }^{* * *} P<0.001$ 


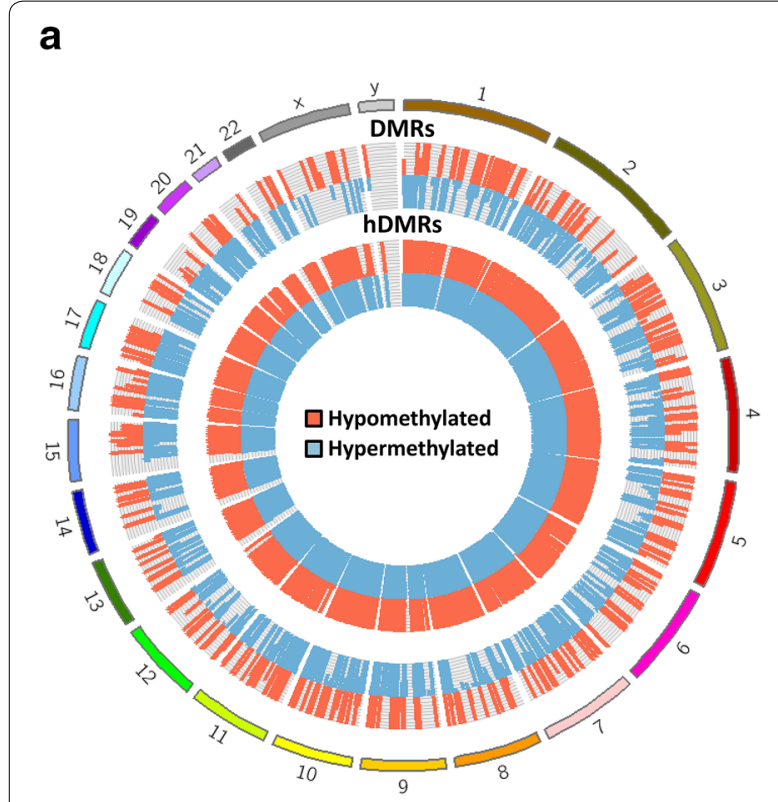

b

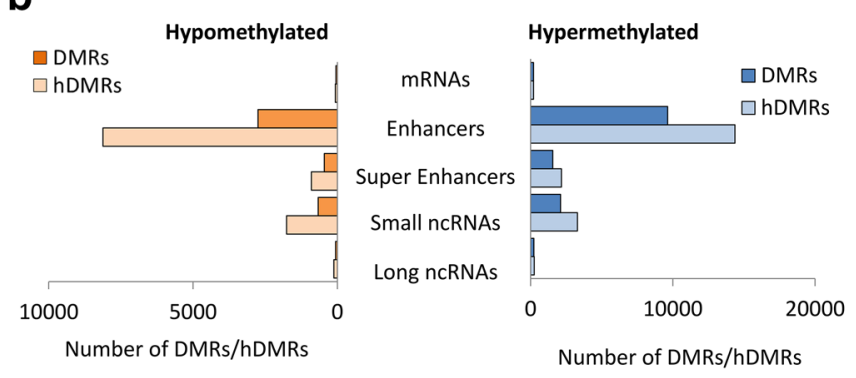

C
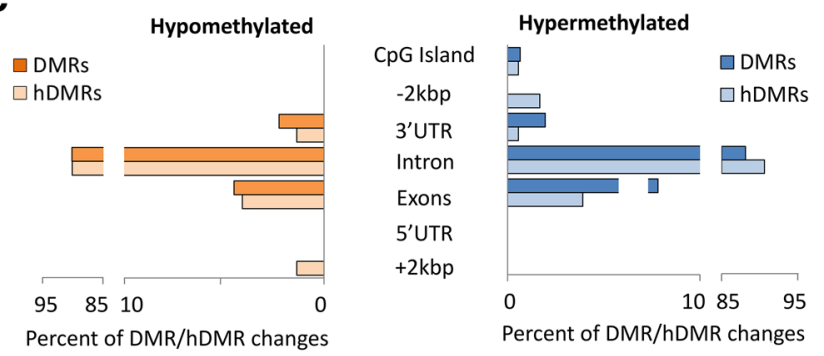

d

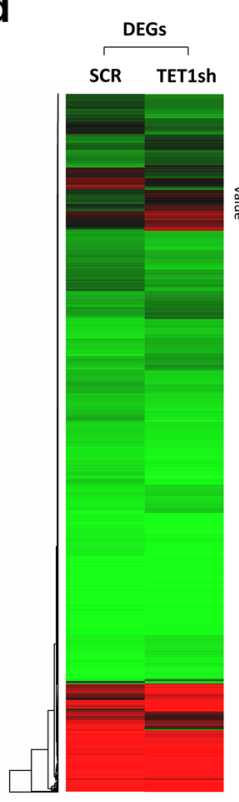

e

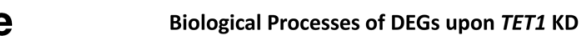

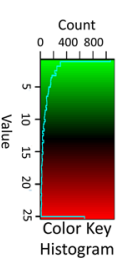
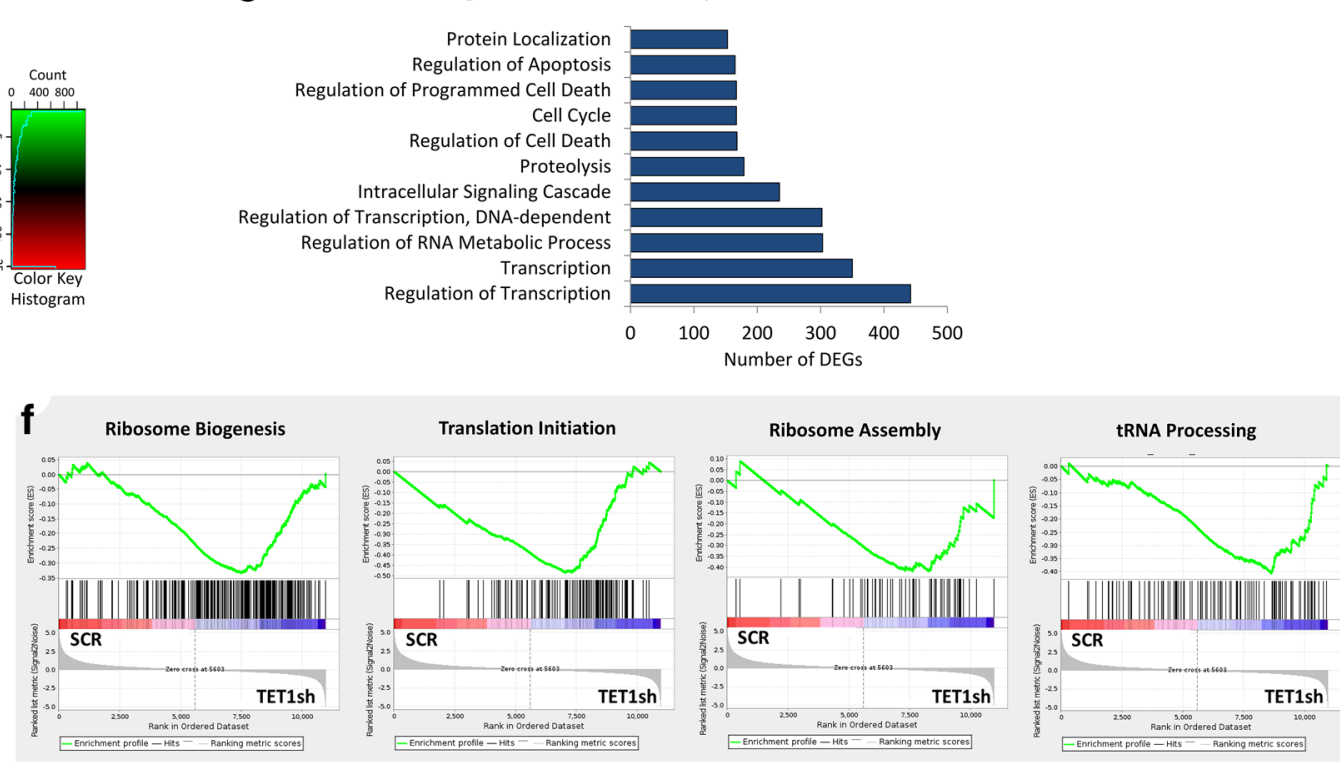

Fig. 5 TET1 KD in human T-ALL cells alters gene expression by changing DNA (hydroxy)methylation. MeDIP-, hMeDIP-, and RNA-seq analysis of human T-ALL cells (CCRF-CEM) before (SCR) and upon TET1 KD (TET1sh). a Genomic distribution of DMRs and hDMRs is displayed as chromosome-based circular plot. Cutoff: $\log 2 \mathrm{FC} \geq 1$ with a $P$ value of $\leq 10^{-4}$. $\mathbf{b}$ Hypo- or hypermethylated DMRs and hDMRs are shown annotated for their association with mRNAs, enhancers, super-enhancers, small ncRNAs, and long ncRNAs. c Hypo- or hypermethylated DMRs and hDMRs associated with mRNAs are shown annotated for cis-regulatory elements: CpG islands, exons, introns, 5'-/3'-UTRs, and sequences greater than $2 \mathrm{kbp}$ upstream or downstream of the nearest gene. $\mathbf{d}$ RNA-seq analysis: heatmap showing hierarchical clustering of 3302 DEGs. Gene names are listed in Additional file 3. e Gene ontology analysis (DAVID) based on DEGs. $\mathbf{f}$ Gene set enrichment analysis (GSEA) of RNA expression associated with biological processes. $\mathbf{g}$ Intersection between DMRs, hDMRs, and DEGs. Gene names are listed in Additional file 3. Heatmap showing hierarchical clustering of genes both differentially expressed and differentially (hydroxy)methylated: $\mathbf{h}$ DEGs and DMRs and $\mathbf{i}$ DEGs and hDMRs 
g

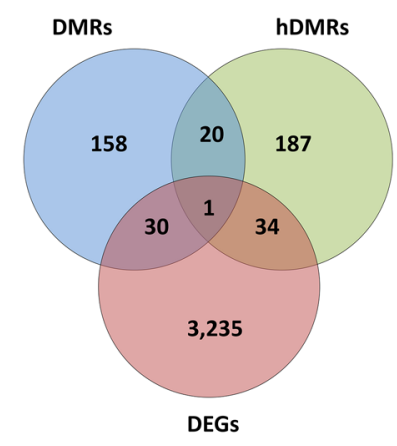

h

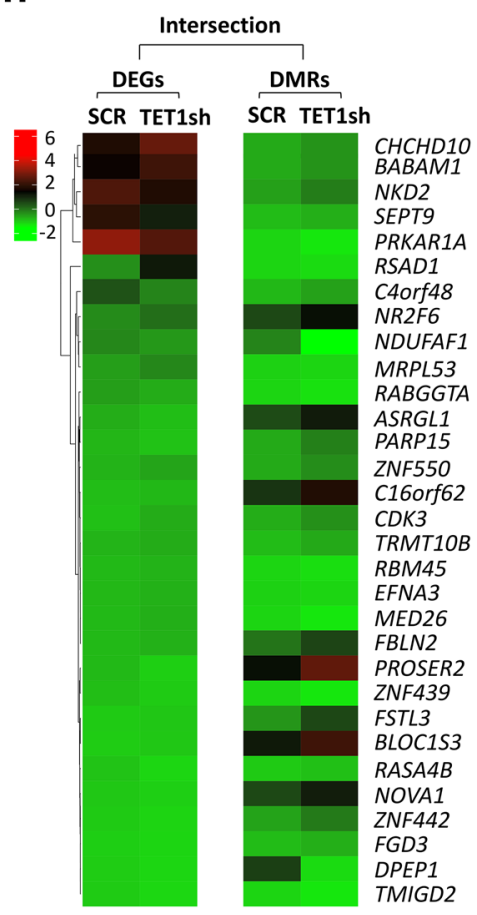

i

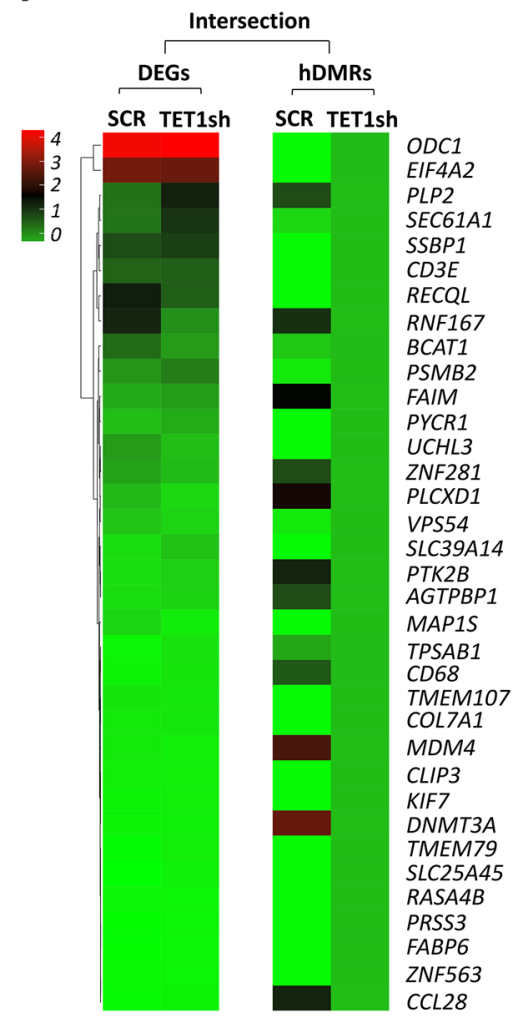

Fig. 5 continued

eliciting cellular senescence and tumor regression [16, 34].

To further determine the relationship between DNA (hydroxy)methylation and gene expression changes in CCRF-CEM cells upon TET1 KD, we selected for DEGs that are associated with changes in $5 \mathrm{mC}$ or 5hmC enrichment. We plotted DMRs, hDMRs, and DEGs in a Venn diagram (Fig. 5g). The intersection graph shows a total of 3300 DEGs, as well as 209 DMRs and 242 hDMRs that are associated with protein coding genes. Of the 3300 DEGs, we identified 31 to be associated with DMRs and 35 with hDMRs (Fig. 5h, i). We found that 21 DMRs and hDMRs overlap with each other. One gene, $R A S A 4 B$ (RAS P21 Protein Activator $4 \mathrm{~B}$ ), was significantly changed in all three categories, DEGs, DMRs, and hDMRs. We conclude that in T-ALL, MYC-driven overexpression of TET1 contributes to tumor cell-specific $5 \mathrm{mC}$ and $5 \mathrm{hmC}$ patterns and thus gene expression programs that are important for enhanced global protein synthesis and tumor cell proliferation.

\section{Ectopic expression of TET2 decreased tumor cell proliferation of human T-ALL cells}

To determine whether reconstitution of TET2 expression in human T-ALL cells affects tumor cell proliferation and viability, we retrovirally introduced full-length TET2 cDNA into CCRF-CEM cells (Fig. 6). RT-qPCR analysis confirmed ectopic TET2 mRNA expression in CCRFCEM (TET2) compared to empty vector (EV) control cells (Fig. 6a), while $M Y C$ expression levels remained unchanged (Fig. 6b). The corresponding growth curve revealed that ectopic TET2 expression significantly decreases proliferation of CCRF-CEM cells (Fig. 6c). To determine the cellular mechanism underlying reduced cell proliferation, we performed flow cytometric cell viability and cell cycle analysis. Cell cycle analysis based on propidium iodide (PI) indicated no significant change in G1, S, or G2/M phase of the cell cycle (Fig. 6d). In parallel, we quantified cell death by Annexin V and PI staining followed by flow cytometric analysis (Fig. 6e). While we did not detect significant changes in the fraction of apoptotic cells, we found a significant increase in necrotic cells (from $1.7 \%$ to $2.6 \%$ ) upon ectopic TET2 expression. We conclude that ectopic TET2 expression in human T-ALL disrupts tumor cell growth and results in reduced cell proliferation primarily through necrosis. 


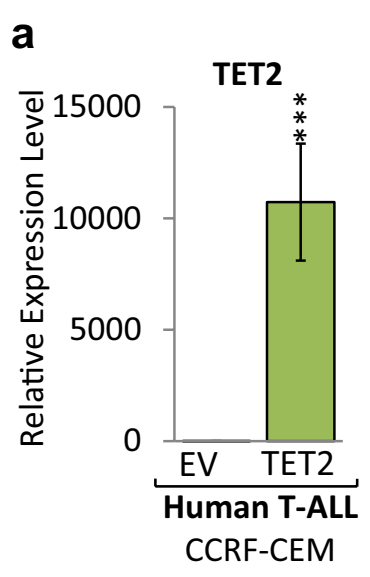

d

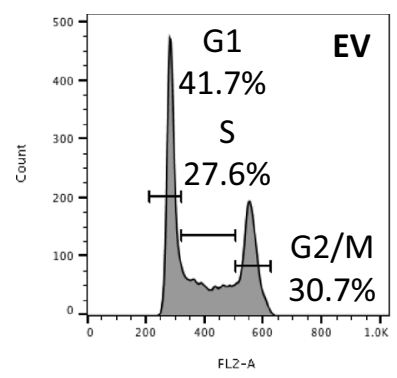

e

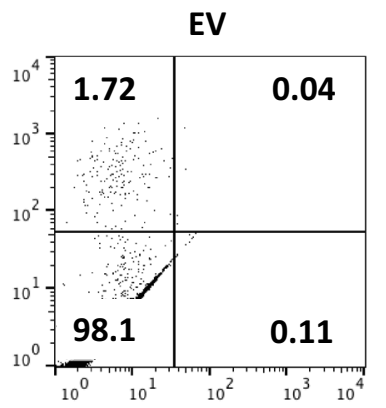

b
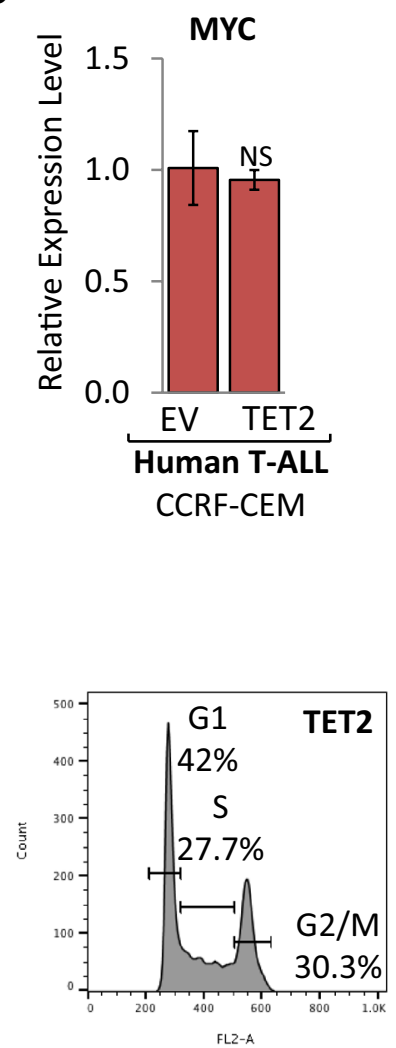

TET2

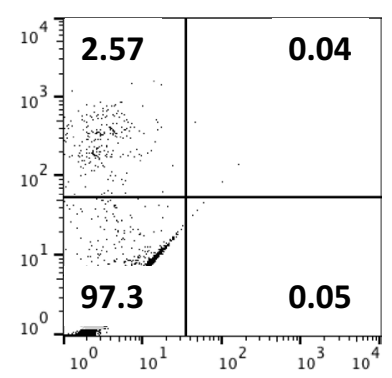

C Human T-ALL CCRF-CEM
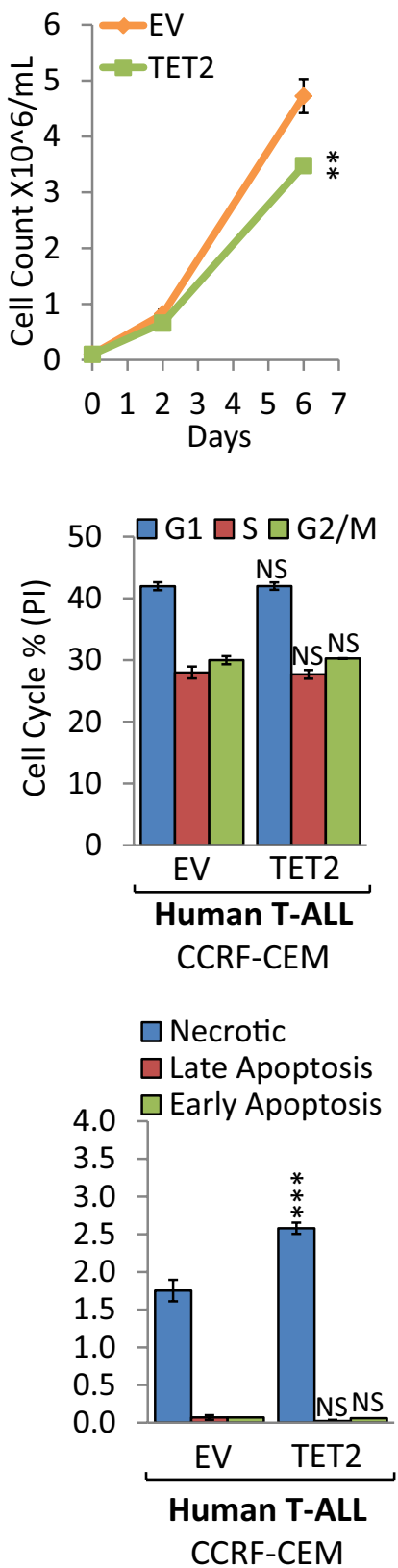

Fig. 6 Ectopic TET2 expression reduces cell proliferation of human T-ALL. Human T-ALL cells (CCRF-CEM), engineered to ectopically express TET2 (TET2), were compared with empty vector (EV) controls. RT-qPCR analysis of a TET2 and $\mathbf{b}$ MYC expression. RT-qPCR data were normalized to RPL13a. c Growth curve comparing viable cell counts. d Flow cytometric cell cycle analysis based on propidium iodide (PI) staining. Cell cycle distribution (G1, S, and G2/M) displayed in percent. e Flow cytometric analysis of apoptosis based on Annexin V/PI staining. Flow cytometry profile of Annexin $V$ staining $(X$ axis) and PI ( $Y$ axis) is shown for representative samples. The lower right quadrant indicates the percentage of early apoptotic cells in each condition; the upper right quadrant indicates the percentage of late apoptotic cells; the upper left quadrant indicates the percentage of necrotic cells. Apoptotic cells (Annexin V-positive cells) are displayed as the percentage of gated cells. Error bars represent $\operatorname{mean} \pm S E M ; n=3$; two-tailed Student's $t$ test: ${ }^{*} P<0.05 ;{ }^{*} P<0.01 ; * * *<<0.001$ 

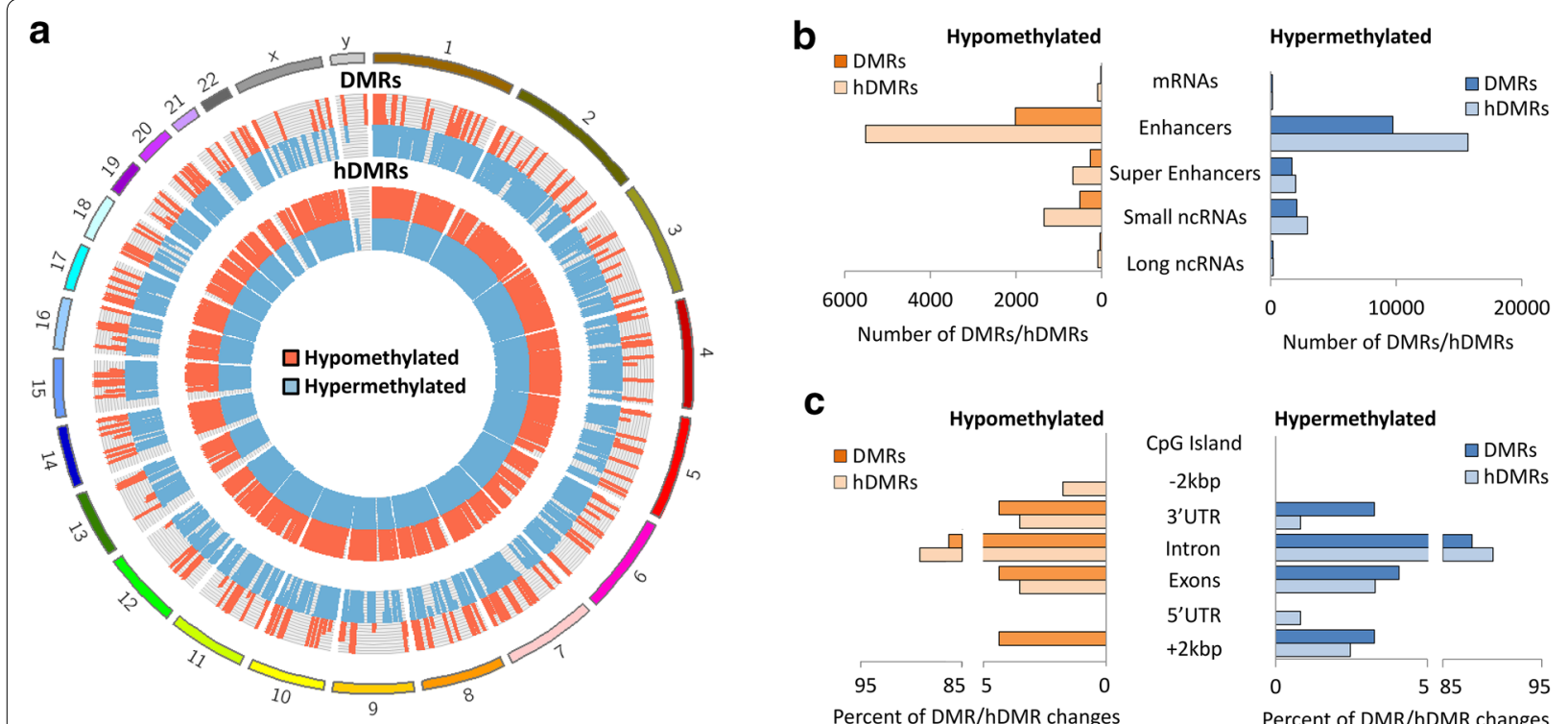

C

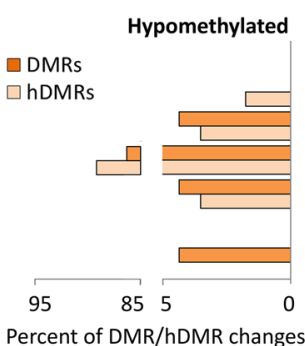
CpG Island
$-2 k b p$
3'UTR
Intron
Exons
$5^{\prime} \mathrm{UTR}$
$+2 \mathrm{kbp}$

Percent of DMR/hDMR changes

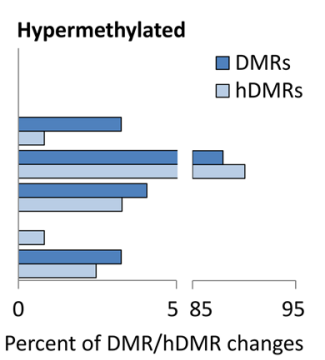

d

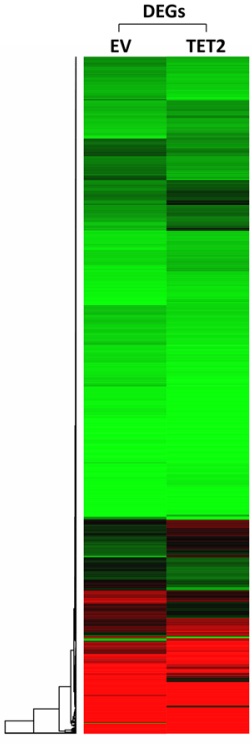

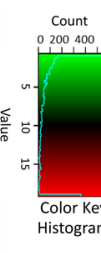

e

Biological Processes of DEGs upon ectopic TET2 expression

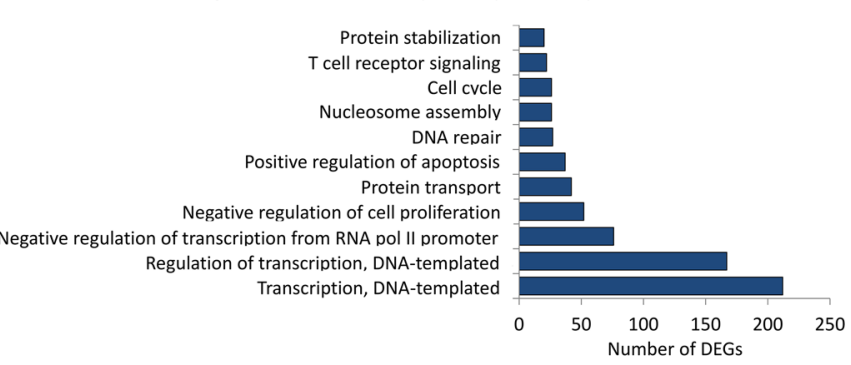

f
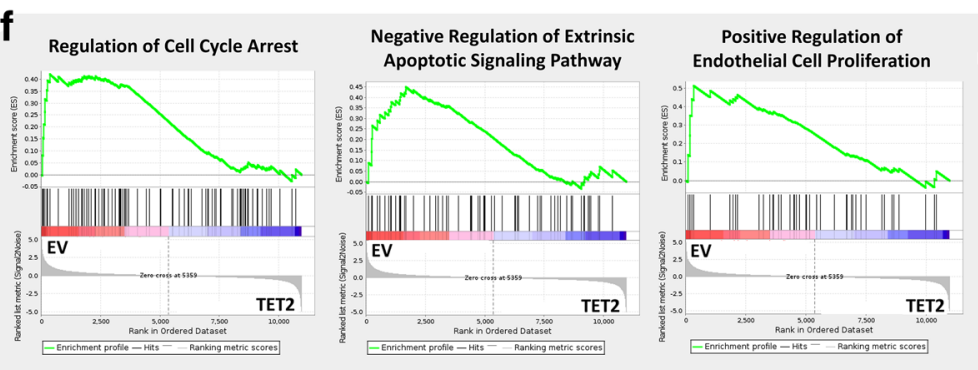

Regulation of Transcription from RNA Poly II Promoter in Response to Stress
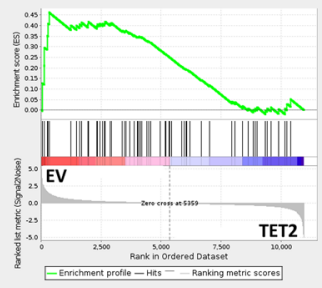

Fig. 7 Ectopic TET2 expression in human T-ALL cells alters gene expression by changing $5 \mathrm{mC}$ and $5 \mathrm{hmC}$ patterns. MeDIP-, hMeDIP-, and RNA-seq analysis of human T-ALL cells (CCRF-CEM) upon ectopic expression of TET2 (TET2) compared to empty vector (EV) control. a Genomic distribution of DMRs and hDMRs is displayed as chromosome-based circular plot. Cutoff: $\log 2 \mathrm{FC} \geq 1$ with a $P$ value of $\leq 10^{-4}$. b Hypo- or hypermethylated DMRs and hDMRs are shown annotated for their association with mRNAs, enhancers, super-enhancers, small ncRNAs, and long ncRNAs. $\mathbf{c}$ Hypo- or hypermethylated DMRs and hDMRs associated with mRNAs are shown annotated for cis-regulatory elements: CpG islands, exons, introns, 5'-/3'-UTRs, and sequences greater than $2 \mathrm{kbp}$ upstream or downstream of the nearest gene. $\mathbf{d}$ RNA-seq analysis: heatmap showing hierarchical clustering of 1771 DEGs. Gene names are listed in Additional file 3. e Gene ontology analysis (DAVID) based on DEGs. f Gene set enrichment analysis (GSEA) of RNA expression associated with biological processes. $\mathbf{g}$ Intersection between DMRs, hDMRs, and DEGs. Gene names are listed in Additional file 3. Heatmap showing hierarchical clustering of genes both differentially expressed and differentially (hydroxy)methylated: $\mathbf{h}$ DEGs and DMRs and $\mathbf{i}$ DEGs and hDMRs 
g

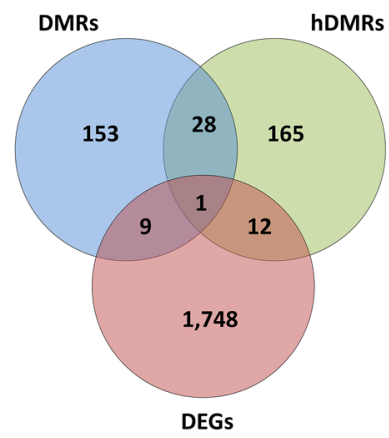

h

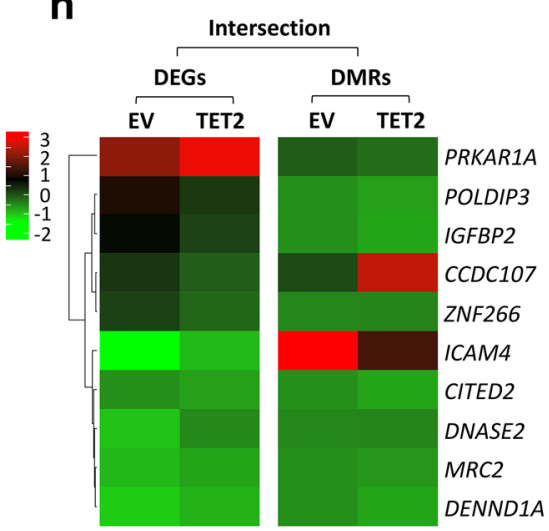

i

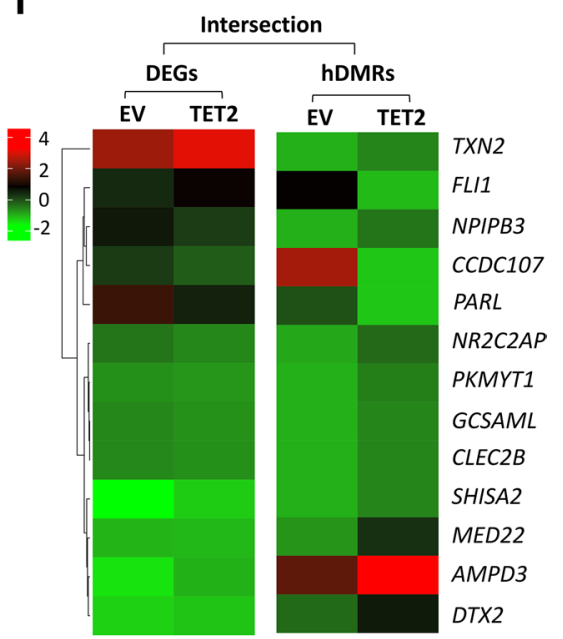

Fig. 7 continued

\section{Ectopic expression of TET2 in human T-ALL cells alters gene expression programs by changing DNA (hydroxy) methylation}

To determine the effect ectopic TET2 expression has on DNA (hydroxy)methylation and RNA expression in human T-ALL cells, we carried out MeDIP-, hMeDIP-, and RNA-seq analysis, comparing CCRF-CEM cells before (EV) and upon ectopic expression of TET2 using cDNA (TET2) (Fig. 7). For each MeDIP/hMeDIP sample, 30-40 million Illumina sequencing reads were generated, of which $\sim 40-85 \%$ were successfully mapped to either strand of the human genome (hg19). To identify DMRs and hDMRs, we performed a genome-wide, unbiased detection by complete tiling of the human genome using a (hydroxy)methylation difference cutoff of $\log 2 \mathrm{FC} \geq 1$ with a $P$ value of $\leq 10^{-4}$.

We identified a total of 16,666 DMRs and 28,681 hDMRs that were significantly hypo- or hypermethylated upon ectopic TET2 expression in CCRF-CEM cells. The genomic location of DMRs and hDMRs indicates genome-wide changes in $5 \mathrm{mC}$ and $5 \mathrm{hmC}$ distribution upon ectopic TET2 expression (Fig. 7a). Annotating DMRs and hDMRs (hypo- and hypermethylated) with the nearest gene, we found 158 and 238 DMRs and hDMRs associated with mRNAs, 11,746 and 21,224 with enhancers, 1957 and 2665 with superenhancers, 2588 and 4272 with small ncRNAs, and 226 and 292 with long ncRNAs, respectively (Fig. 7b). We further annotated DMRs and hDMRs associated with mRNA genes with cis-elements. Of the total DMRs and hDMRs, we found $86.96 \%$ and $91.93 \%$ associated with introns, $4.21 \%$ and $3.39 \%$ with exons, $3.80 \%$ and $2.57 \%$ with $5^{\prime}$ - or $3^{\prime}$-UTRs, and $3.80 \%$ and $2.11 \%$ with sequences $2 \mathrm{kbp}$ or more upstream or downstream of the nearest gene, respectively (Fig. $7 \mathrm{c}$ ). Thus, similar to TET1 KD, ectopic expression of TET2 in human T-ALL cells led to genome-wide changes in $5 \mathrm{mC}$ and $5 \mathrm{hmC}$ patterns associated mostly with intron sequences.

To determine the effect of alterations in DNA (hydroxy) methylation upon ectopic TET2 expression on RNA expression in CCRF-CEM cells, we next performed RNA-seq. We identified 1771 statistically significant DEGs, of which 917 genes decreased, while 854 increased in expression upon ectopic TET2 expression (Fig. 7d). To determine the biological processes associated with the DEGs, we performed gene ontology analysis using DAVID (Fig. 7e). We found regulation of transcription, negative regulation of cell proliferation, and positive regulation of apoptosis among the processes associated with ectopic TET2 expression in T-ALL. We further performed gene enrichment analysis to associate RNA expression with biological processes. The results indicate that upon ectopic TET2 expression, there is a decreasing trend for genes involved in cell cycle arrest $(n=84)$, negative regulation of extrinsic apoptotic signaling $(n=66)$, positive regulation of endothelial cell proliferation $(n=77)$, and regulation of transcription from RNA Pol II promoter in response to stress $(n=58)$ (Fig. $7 f)$.

To determine the relationship between DNA (hydroxy) methylation and gene expression changes in CCRFCEM cells upon ectopic TET2 expression, we selected for DEGs that are associated with changes in $5 \mathrm{mC}$ or 5hmC enrichment. We plotted DMRs and hDMRs associated with mRNAs, as well as DEGs in a Venn diagram (Fig. 7g). The intersection graph shows a total of 1771 DEGs, as well as 150 DMRs and 200 hDMRs that are associated with protein-coding genes. Of the 1771 DEGs, we identified 10 to be associated with DMRs and 13 with 


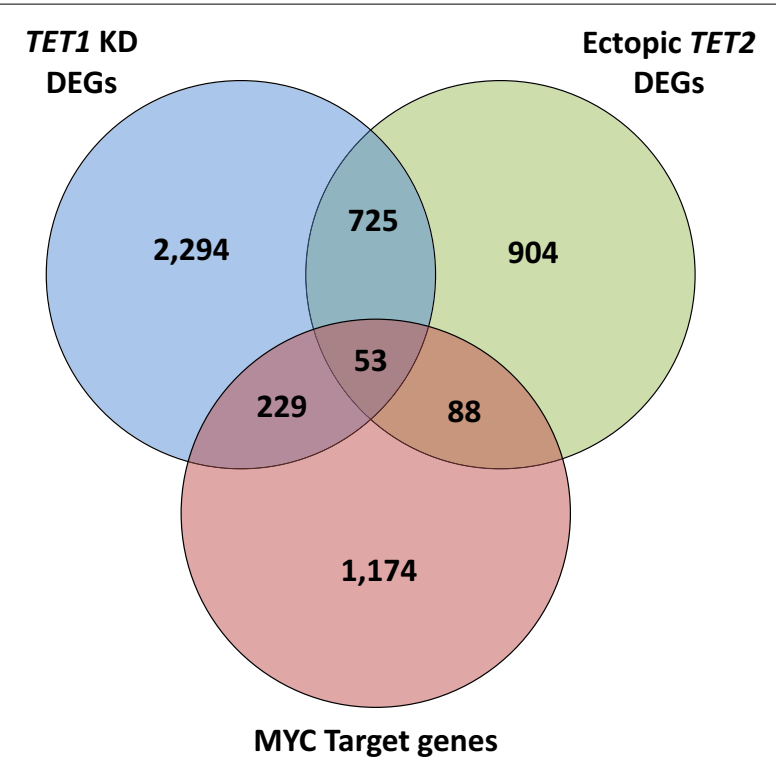

Fig. 8 TET1, TET2, and MYC target genes overlap. Overlap between DEGs upon TET1 KD and ectopic TET2 expression in human T-ALL cells (CCRF-CEM), and previously identified MYC target genes, obtained from the Molecular Signatures Database (MSigDB) [35-37]. The intersection between genes is displayed as Venn diagram. Gene names are listed in Additional file 3

hDMRs (Fig. 7h, i). We found that 25 DMR- and hDMRassociated protein-coding genes overlap. CCDC107 (coiled-coil domain-containing protein 107), was significantly changed in all three categories: DEGs, DMRs, and hDMRs. Taken together, we conclude that ectopic expression of TET2 in T-ALL alters $5 \mathrm{mC}$ and $5 \mathrm{hmC}$ patterns and thus gene expression programs resulting in reduced tumor cell proliferation.

\section{TET1, TET2, and MYC target genes overlap}

To determine the overlap between TET1, TET2, and MYC target genes in T-ALL, we used the DEGs upon TET1 KD and ectopic expression of TET2 in human T-ALL cells (CCRF-CEM) and compared them with previously identified MYC target genes [35-37] (Fig. 8). Of the 3300 DEGs identified upon TET1 KD and the 1771 DEGs identified upon ectopic expression of TET2, we found 778 genes overlap with each other. At the same time, we found 282 genes that were both regulated by TET1 and MYC, and 141 genes that were both regulated between TET2 and MYC. Lastly, we found 53 genes that all three data sets had in common. Taken together, this indicates that there is some overlap between TET1 and TET2 targets, even though the two enzymes have distinct functions in T-ALL. At the same time, fewer TET1 and TET2 targets overlap with MYC, revealing that the majority of TET targets are not directly occupied by
MYC, but rather regulated indirectly highlighting an indirect genome-wide mechanism.

\section{Discussion}

This study demonstrates for the first time that the MYC oncogene deregulates the expression of TET methylcytosine dioxygenases and thereby global DNA (hydroxy) methylation and gene expression programs to maintain tumor cell proliferation. While aberrant DNA methylation is a characteristic feature of tumor cells, the mechanisms of how tumor cell-specific DNA (hydroxy) methylation patterns are written, maintained, and erased through the coordinated action of DNA methylating and demethylating enzymes are poorly understood. Here, we reveal a novel mechanism through which MYC establishes and maintains tumor cell-specific DNA (hydroxy) methylation and gene expression programs in a genomewide fashion.

By carrying out a comprehensive genome-wide DNA (hydroxy)methylation analysis, we show that cellular senescence and tumor regression upon MYC inactivation

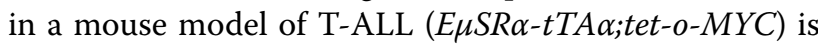
associated with genome-wide changes in $5 \mathrm{mC}$ and $5 \mathrm{hmC}$ patterns. Using this T-ALL model, we have previously reported that tumor regression depends on activation of cellular senescence pathways associated with genomewide changes in chromatin structure including histone acetylation and methylation, associated with heterochromatin formation [3, 18, 30]. Together, our MeDIP- and hMeDIP-seq analyses reveal genome-wide changes in $5 \mathrm{mC}$ and $5 \mathrm{hmC}$ patterns associated with a wide variety of biological processes upon MYC inactivation, cellular senescence, and tumor regression, indicating that MYC maintains tumor cell-specific DNA (hydroxy)methylation patterns in T-ALL.

Shedding light on the underlying molecular mechanism, we found that in T-ALL, TET1 is overexpressed, while TET2 transcription is repressed in a MYC-dependent fashion across all human T-ALL cell lines and clinical specimens we analyzed. Consistently, we were able to demonstrate that MYC inactivation in T-ALL arising in $E \mu S R \alpha-t T A$;tet-o-MYC mice leads to an inverse expression pattern, decreasing TET1 levels, while increasing TET2 levels. Together with the ChIP data indicating that MYC binds to the TET1 and TET2 loci, our results implicate a direct transcriptional regulation. However, we cannot exclude that, besides being controlled by MYC directly, TET1 and TET2 expression might also be regulated indirectly through changes in other MYC target genes. Both DNMT and TET expression and activity have been found deregulated in various cancer types, including hematologic malignancies [38-40]. We conclude that the MYC oncogene upregulates TET1 while suppressing 
TET2 expression in T-ALL, and speculated whether TET function was essential for tumor maintenance. Similarly, we previously reported that in T-ALL and Burkitt lymphoma MYC directly controls the overexpression of $D N M T 3 B$ for tumor maintenance, maintaining specific $5 \mathrm{mC}$ and thus gene expression patterns [26].

Indeed, we found that TET1 KD or ectopic expression of TET2 decreased cell proliferation in T-ALL and was associated with genome-wide changes in $5 \mathrm{mC}$ and $5 \mathrm{hmC}$, suggesting a tumor promoting function of TET1, and a tumor suppressing role for TET2. Our findings are consistent with recent reports that TET1 acts as an oncogene in acute myeloid leukemia (AML) development and that high TET1 levels are predictive of poor overall survival in AML [41]. Oncogenic TET1 plays an important role in the development of MLL-rearranged leukemia [42-44]. TET1 is also overexpressed in $40 \%$ of patients with triple-negative breast cancer, where it is associated with DNA hypomethylation and activation of oncogenic pathways, leading to poor overall survival [45]. However, TET1 expression has been found low in many other solid tumor types including colon, gastric, and ER-negative breast cancer [46-49], where TET1 is downregulated through miR-29, HMGA2, or NF-кB activation [50, 51]. Thus, TET1 may act as either tumor promotor or suppressor dependent on the context, a notion that also has been reported for other epigenetic regulators, including DNMTs.

In T-ALL, we found loss of TET1 function to be associated with decreased ribosome biogenesis and assembly, translational regulation, and tRNA processing. Indeed, MYC is well known to regulate ribosome biogenesis and translation through multiple mechanisms (reviewed in [52]), and we previously demonstrated that MYC inactivation leads to shutdown of global protein synthesis resulting in cellular senescence and tumor regression $[16$, 34]. Our finding that in T-ALL loss of TET1 expression is linked to a decrease in ribosome biogenesis and translational regulation might provide a novel mechanism of how MYC regulates protein synthesis through alterations in DNA (hydroxy)methylation patterns. Consistent with our findings in T-ALL, high TET1 expression also correlated with upregulation of RNA transport and ribosome biogenesis pathways in AML [41]. In contrast to TET1, our results show that TET2 acts as a tumor suppressor in T-ALL. We found ectopic expressing of TET2 to be associated with a decrease in cell cycle regulation, negative regulation of apoptosis, and positive regulation of cell proliferation. While the absence of TET2 in MYC-driven tumors does not automatically mean it reinforces the tumor state, our ectopic TET2 expression experiments indicate a role as tumor suppressor. Similarly, TET2 has been reported to be a tumor suppressor in B-cell lymphomagenesis [53].

TET1 KD and ectopic TET2 expression in T-ALL causes genome-wide changes in both $5 \mathrm{mC}$ and $5 \mathrm{hmC}$ patterns. TET1 KD in T-ALL causes differential hydroxymethylation at KLF4, critical for regulation of proliferation, differentiation, apoptosis, and somatic cell reprogramming [54]. There are several TET2 targets in T-ALL that overlap with AML, such as CCDC84, CHKA, GNGT2, GPS2, IL20RB, LIN7B, PPAN, PROCA1, RECQL4, TMSB10, $C H K B$ (fusion), and PABPN1 (fusion). It is noteworthy that restoration of TET2 in AML promotes DNA demethylation, cell differentiation, and cell death, leading to a block in self-renewal of hematopoietic stem cells, causing myeloid differentiation [55].

While the exact role of TET1 and TET2 in regulating DNA (hydroxy)methylation outside of developmental processes is not well understood, our findings indicate distinct functions of TET1 and TET2 in MYC-driven tumor maintenance. In our working model (Fig. 9), the MYC oncogene directly drives the transcription of TET1 (as well as DNMT1 and DNMT3B [26]), while suppressing TET2. Inactivation of MYC inverts the expression pattern of both DNMTs and TETs, eliciting cellular senescence and tumor regression. Loss of TET1 and overexpression of TET2 cause genomewide changes in $5 \mathrm{mC}$ and $5 \mathrm{hmC}$ patterns. We found a majority of changes in DNA (hydroxy)methylation patterns occurred in intron sequences. These observations are consistent with other studies, indicating that TET1 localizes to gene bodies and introns of a large number of genes [56-58]. Our data suggest that TET1 and TET2 have distinct sets of genomic targets in turn affecting distinct cellular processes, despite some overlap in differentially expressed genes. In this regard, it would be of interest to uncover which of the differentially (hydroxy)methylated genes are directly bound by TET 1 or TET 2 in T-ALL using genome-wide location analysis. Furthermore, it would be interesting to determine whether the genomic targets of DNMT1 and DNMT3B in this context overlap with TET1 or TET2, and whether there is any synergistic effect between the components of the DNA methylating and demethylating enzymes contributing to MYC-driven tumor maintenance.

\section{Conclusions}

This study provides novel evidence that MYC directly deregulates the expression of TET1 and TET2 in T-ALL to maintain $5 \mathrm{mC}$ and $5 \mathrm{hmC}$ patterns in a genome-wide fashion, which is associated with tumor cell-specific gene expression. Our results reveal that the MYC oncogene 


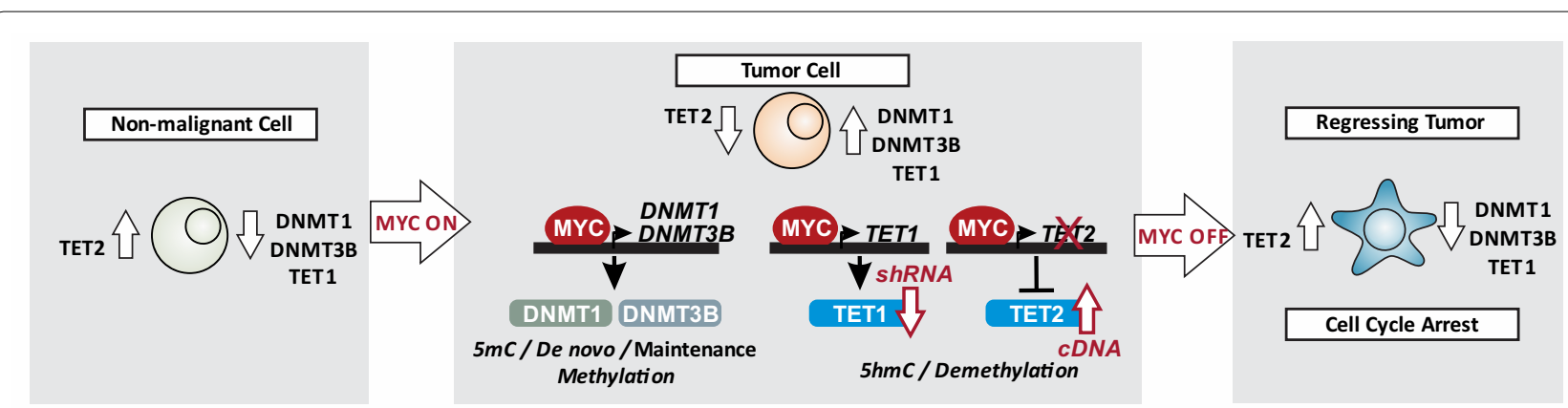

Fig. 9 Working model: The MYC oncogene controls DNMT1 and DNMT3B, as well as TET1 and TET2 expression in T-ALL. Non-malignant cells: MYC levels are low, corresponding with low TET1, as well as DNMT1 and DNMT3B expression. Tumor cells: MYC levels are constitutively high, driving the expression of TET1 as well as DNMT1 and DNMT3B, while suppressing TET2. Regressing tumor: MYC inactivation in T-ALL causes tumor regression through cellular senescence. This is associated with diminished DNMT1, DNMT3B, and TET1 levels, while TET2 expression is increased. LOSS of TET1 function (shRNA) and reconstitution of TET2 expression (CDNA) led to broad changes in $5 \mathrm{mC}$ and $5 \mathrm{hmC}$ patterns, in turn affecting a variety of cellular processes causing reduced tumor cell proliferation. Taken together, MYC induces and maintains a tumor cell-specific global $5 \mathrm{mC}$ and $5 \mathrm{hmC}$ patterns through control of DNMT and TET expression

establishes and maintains tumor cell-specific DNA (hydroxy)methylation patterns on a genome-wide level by modulating the expression of individual components of the DNA methylating and demethylating machinery. Our working model indicates a coordinated interplay between the components of the DNA methylating and demethylating machinery contributing to MYC-driven tumor maintenance, highlighting the potential of specific TET enzymes for therapeutic strategies. Targeting DNMTs or TET enzymes pharmacologically for therapeutic anticancer strategies may be a promising concept, even though specific small molecule inhibitors are currently still elusive.

\section{Methods}

\section{Cell culture and treatment}

Mouse T-ALL cell lines, derived from the transgenic

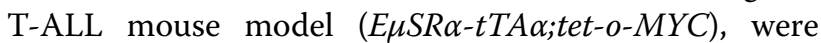
kindly provided by Dr. Dean W. Felsher, Stanford University [5]. Human Burkitt lymphoma-like cells (P4936) [59], $\mathrm{T}$ cell leukemia/lymphoma cell lines (JURKAT, CCRF-CEM, MOLT3, MOLT4, and CCRF-HSB-2), and adherent packaging cell lines (HEK293T and Phoenixampho) were obtained from American Type Culture Collection (ATCC). To turn off expression of the tetracycline-regulated MYC in the mouse model and in P493-6 cells, $20 \mathrm{ng} / \mathrm{mL}$ doxycycline (DOX) was added to the cell culture medium for the indicated times. All leukemia/ lymphoma cell lines were passaged less than 8 times, maintained in RPMI1640 supplemented with 10\% FBS, $1 \%$ penicillin/streptomycin, $1 \%$ L-glutamine, and $50 \mu \mathrm{M}$ 2-mercaptoethanol, and incubated at $37{ }^{\circ} \mathrm{C}$ humidified with $5 \% \mathrm{CO}_{2}$. Adherent cell lines were passaged less than 8 times, maintained in DMEM supplemented with $10 \%$ FBS, $1 \%$ penicillin/streptomycin, $1 \%$ L-glutamine, and $50 \mu \mathrm{M}$ 2-mercaptoethanol, and incubated at $37{ }^{\circ} \mathrm{C}$ humidified with $5 \% \mathrm{CO}_{2}$. Routine mycoplasma detection is done on all cell lines to eliminate risk of contamination.

\section{shRNA-mediated knockdown}

Cell lines were infected with lentiviral vectors (pLKO.1puro) containing either scrambled control (SCR) or specific shRNA directed against TET1. The specific oligo sequences of shRNA are: Hs. TET1sh CCGGACACA ACTTGCTTCGATAATTCTCGAGAATTATCGA AGC AAGTTGTGTTTTTTG and Mm. TET1sh CCGGCA ACTTGCATCCACGATTAATCTCGAGATTAATCG TGGATGCAAGTTGTTTTTG. Briefly, HEK293T cells were transfected using Lipofectamine 2000 (Invitrogen) with pLKO.1-puro, pPAX2, and pMD.G plasmids. Virus particles were collected for spinoculation at $2400 \mathrm{rpm}$ for $2 \mathrm{~h}$ at $32{ }^{\circ} \mathrm{C}$. Upon selection of positive cells with $2-4 \mu \mathrm{g}$ of puromycin, knockdown of TET1 was confirmed by RT-qPCR.

\section{Ectopic gene expression}

Cell lines were infected with retroviral vectors (pMSCVPIG) containing either empty vector (EV) or a mouse TET2 full-length cDNA. pcDNA3-Tet2 (\#60939) and MSCV PIG (Puro-IRES GFP) (\#18751) were obtained from Addgene. Mm TET2 cDNA was subcloned from pCDNA3-Tet2 using SnaBI/NotI restriction sites, into adapted pMSCV-PIG (Puro-IRES-GFP) plasmid using $\mathrm{HpaI} / \mathrm{NotI}$ restriction sites. Adapted pMSCV-PIG plasmid vector was modified with destroyed second EcoRI site and introduced NotI site and was a gift from Dr. Honglin Li, Augusta University. pMSCV-PIG-MmTET2 
clone was verified via DNA sequencing. Briefly, PhoenixAmpho cells were transfected using Lipofectamine 2000 (Invitrogen) with pMSCV-PIG plasmid. Virus particles were collected for spinoculation at $2400 \mathrm{rpm}$ for $2 \mathrm{~h}$ at $32{ }^{\circ} \mathrm{C}$. Upon selection of positive cells with $2-4 \mu \mathrm{g}$ of puromycin, cDNA expression was detected using RTqPCR for TET2.

\section{Tissue collection}

Human spleen (total RNA) obtained from a healthy donor was purchased from Zyagen Inc. Human PBMCs (total RNA) were obtained from the Augusta University Biorepository.

\section{RNA extraction and analysis of gene expression}

Total RNA was isolated using the NucleoSpin RNA Kit including DNase-I digest (Machery-Nagel Inc.) following the manufacturer's protocol. $0.5 \mu \mathrm{g}$ RNA was reverse transcribed into cDNA using the iScript cDNA Kit (BioRad). Quantitative PCR ( $\mathrm{qPCR}$ ) was performed using iTAQ Universal SYBR GREEN (BioRad) in an ABI StepOne Plus analyzer (Applied Biosystems). Specific primer sequences are as follows: $M m M Y C \mathrm{~F}$ : TCTCCATCCTAT GTTGCGGTC, R: TCCAAGTAACTCGGTCATCATCT; $M m$ ODC1 F: GACGAGTTTGACTGCCACATC, R: CGCAACATAGAACGCATCCTT; $M m$ TET1 F: ATT TCCGCATCTGGGAACCTG, R: GGAAGTTGATCT TTGGGGCAAT; $M m$ TET2 F: TGCTTTCCCAACACG GAACTA, R: GCACCATTAGGCATTAGCACAAT; $M m$ TET3 F: TGCGATTGTGTCGAACAAATAGT, R: TCC ATACCGATCCTCCATGAG; $M m U B C$ F: AGCCCA GTGTTACCACCAAG, R: ACCCAAGAACAAGCA CAAGG; $H s$ MYC F: CTGCGACGAGGAGGAGAA, R: GGCAGCAGCTCGAATTTCTT; Hs TET1 F: CATCAG TCAAGACTTTAAGCCCT, R: CGGGTGGTTTAG GTTCTGTTT; Hs TET2 F: GATAGAACCAACCAT GTTGAGGG, R: TGGAGCTTTGTAGCCAGAGGT; $\mathrm{Hs}$ TET3 F: TCCAGCAACTCCTAGAACTGAG, R: AGG CCGCTTGAATACTGACTG; Hs RPL13A F: CGGACC GTGCGAGGTAT, R: CACCATCCGCTTTTTCTTGTC.

\section{RNA sequencing}

Total RNA was extracted as described above, and samples were quantified using Nanodrop and qualified by agarose gel electrophoresis. Briefly, mRNA was isolated from total RNA with NEBNext PolyA mRNA Magnetic Isolation Module. Alternatively, rRNA was removed from the total RNA with a RiboZero Magnetic Gold Kit. The enriched mRNA or rRNA-depleted RNA was used for RNA-seq library preparation using KAPA Stranded RNA-Seq Library Prep Kit (Illumina). The completed libraries were qualified on Agilent 2100 Bioanalyzer for concentration, fragment size distribution between 400 and $600 \mathrm{bp}$, and adapter dimer contamination. The DNA fragments in mixed libraries were denatured with $0.1 \mathrm{M}$ $\mathrm{NaOH}$ to generate single-stranded DNA molecules, loaded onto channels of the flow cell at $8 \mathrm{pM}$ concentration, and amplified in situ using TruSeq SRCluster Kit v3-cBot-HS (\#GD-401-3001, Illumina). Sequencing was carried out by running 150 cycles with paired-end reads, using the Illumina HiSeq 4000 according to the manufacturer's instructions. RNA sequencing was performed by Arraystar Inc. (Rockville, MD).

\section{RNA-seq data analysis}

After quality control, the fragments were $5^{\prime}, 3^{\prime}$-adaptortrimmed and filtered $\leq 20$ bp reads with cutadapt software. The trimmed reads were aligned to reference genome with Hisat 2 software [60]. The expression level (FPKM value) of known genes and transcripts were calculated using ballgown through the transcript abundances estimated with StringTie [61, 62]. The number of identified genes and transcripts per group was calculated based on the mean of FPKM in group $\geq 0.5$. Principal component analysis (PCA), correlation analysis, hierarchical clustering, gene ontology (GO), pathway analysis, scatter plots, and volcano plots are performed for the differentially expressed genes in $\mathrm{R}$ or Python environment for statistical computing and graphics. RNA sequencing data analysis was performed by Arraystar Inc. (Rockville, MD). Data sets are deposited in GEO under accession number GSE126029.

\section{Cell cycle analysis using propidium iodide}

Cells were fixed in $70 \%$ methanol at $-20{ }^{\circ} \mathrm{C}$ for a minimum of $72 \mathrm{~h}$ and stained using a propidium iodide (PI) solution containing PBS $+0.5 \%$ BSA, $50 \mu \mathrm{g} / \mathrm{mL}$ PI (Acros Organics), and $200 \mu \mathrm{g} / \mathrm{mL}$ RNase A (Thermo Fisher). Cells were then analyzed immediately on a FACScalibur flow cytometer (Becton-Dickinson). FACS data were analyzed using FlowJo software (Tree Star).

\section{Apoptosis analysis using Annexin V/propidium iodide}

Annexin V-FITC and PI staining was used for the study of cell cycle distribution and apoptosis using the Annexin V-FITC Early Apoptosis Detection Kit (Cell Signaling). Briefly, cells were washed in PBS and suspended in 1X Annexin V binding buffer. Annexin V-FITC conjugate and propidium iodide were incubated for $10 \mathrm{~min}$ on ice and immediately analyzed on a FACScalibur flow cytometer (Becton-Dickinson). FACS data were analyzed using FlowJo software (Tree Star). 


\section{MeDIP and hMeDIP sequencing}

Genomic DNA was isolated using the Nucleospin Tissue Kit with RNase A (Macherey-Nagel Inc.). Purified genomic DNA was sonicated to 200-800 bp fragments, and $1 \mu \mathrm{g}$ of fragmented DNA was ligated to Illumina's genomic adapters with Genomic DNA Sample Kit (\#FC-102-1002, Illumina), following the manufacturer's instructions. 300-900 bp ligated DNA fragments were further immunoprecipitated using an anti-5-Methylcytosine antibody (for MeDIP) or an anti-5-Hydroxymethylcytosine antibody (for hMeDIP). The enriched DNA was amplified by PCR and purified by AMPure XP beads. The DNA fragments in mixed libraries were denatured with $0.1 \mathrm{M} \mathrm{NaOH}$ to generate single-stranded DNA molecules, loaded onto channels of the flow cell at 8 pM concentration, and amplified in situ using HiSeq 3000/4000 PE Cluster Kit (\#PE-410-1001, Illumina). Sequencing was carried out by running 150 cycles with paired-end reads, using HiSeq 3000/4000 SBS Kit (\#FC-410-1003, Illumina) on Illumina HiSeq 4000 according to the manufacturer's instructions. MeDIP and hMeDIP sequencing was performed by Arraystar Inc. (Rockville, MD).

\section{MeDIP- and $h$ MeDIP-seq data analysis}

Raw sequencing data generated from Illumina HiSeq 4000 that pass the Illumina chastity filter are used for following analysis. The fragments were $5^{\prime}, 3^{\prime}$-adaptortrimmed and filtered $\leq 16$ bp reads with cutadapt software. Trimmed reads (trimmed $5^{\prime}, 3^{\prime}$-adaptor bases) are mapped to reference genome (hg19 or mm10) with Hisat2 software. The mapped reads were used for statistically significant unbiased methylation/hydroxymethylation region detection. LncRNA-, mRNA-, and small ncRNA-associated DMRs- and hDMRs-enriched regions (peaks) with statistically significant were identified by diffReps (cutoff: $\log 2 \mathrm{FC} \geq 1, P$ value $\leq 10^{-4}$ ) [63]. LncRNA-, mRNA-, and small ncRNA-associated DMRs and hDMRs within promoter were annotated by the nearest gene using the UCSC RefSeq database. Promoter peaks were defined as 2000 bp upstream and downstream from the transcription start site (TSS); gene body peaks were defined as $+2000 \mathrm{bp}$ downstream of the transcription start site (TSS) to the transcription termination site (TTS); and intergenic peaks were defined as the other genomic regions not included in either promoters or gene body. MeDIP and hMeDIP analysis was performed by Arraystar Inc. (Rockville, MD). Data sets are deposited in GEO under accession number GSE126029.

\section{Gene set enrichment analysis (GSEA)}

GSEA (version 2.2.4) was performed using Java-based software (http://software.broadinstitute.org/gsea/ index.jsp) to distinguish important biological processes pathways enriched between two samples [36]. Enrichment score was calculated for gene sets within a pathway and was selected based on nominal $P \leq 0.05$.

\section{Functional annotation of biological processes}

Database for Annotation, Visualization and Integrated Discovery (DAVID) (version 6.8) analysis was performed based on online software (https://david.ncifcrf.gov/ home.jsp) [64]. Gene ontology (GO) analysis of biological processes was performed to indicate genes expressed differentially in biological pathways between two samples. Statistical parameters were based on default DAVID parameters including a threshold count of 2 with EASE of $0.1, P$ value $\leq 0.05$, and Benjamini threshold $\leq 1$.

\section{Clinical specimen data analysis}

Gene expression analysis in clinical specimens was performed using Oncomine (http://www.oncomine.org) data sets.

\section{Statistical analysis}

All experiments were performed on biological replicates unless otherwise specified. Sample size is reported in the respective figure legends. All quantitative PCR were run in triplicates, and standard deviation is shown in the figures. Two-tailed unpaired student's $t$ test was used to calculate $P$ values; statistically significant values are specified in the figure legends. Statistical significance: NS $=$ not significant ${ }^{*} P<0.05,{ }^{* *} P<0.01,{ }^{* * *} P<0.001$.

\section{Additional files}

Additional file 1: Fig. S1. MYC inactivation in T-ALL. T-ALL cells (6780) derived from EMSRa-tTAa;tet-o-MYC mice were treated with $20 \mathrm{ng} / \mathrm{mL}$ DOX for 2 days. RT-qPCR analysis of MYC. RT-qPCR data were normalized to $U B C$. Error bars represent mean $\pm S E M ; n=3$; two-tailed Student's $t$ test: ***P $P<0.001$

Additional file 2: Fig. S2. TET1 knockdown reduces cell proliferation of mouse T-ALL cell lines. Mouse T-ALL cells (EMSRa-tTAa;tet-o-MYC) were compared before (SCR) and upon TET1 KD (TET1sh). RT-qPCR analysis of a MYC and TET1, b TET2 and TET3. RT-qPCR data were normalized to UBC. c Growth curve comparing viable cell counts. d Flow cytometric cell cycle analysis based on propidium iodide (PI) staining. The cell cycle distribution $(G 1, S$, and G2/M) is displayed in percent. Error bars represent mean $\pm S E M$ $n=3$; two-tailed Student's $t$ test: ${ }^{*} P<0.05$; ${ }^{* *} P<0.01$; ${ }^{* * *} P<0.001$.

Additional file 3. Detailed lists of genes used in DMR, hDMR, DEG, GSEA, and intersection analysis. List of genes associated with DMRs and hDMRs in mouse T-ALL cells (EMSRa-tTAa;tet-o-MYC) (6780) before and upon 2 days of MYC inactivation (+DOX). List of genes associated with DMRs and hDMRs, as well as DEGs and intersection analysis in human T-ALL (CCRFCEM) upon TET1 KD. List of genes associated with DMRs and hDMRs, as well as DEGs and intersection analysis in human T-ALL (CCRF-CEM) upon ectopic TET2 expression. List of genes from GSEA analysis in human T-ALL (CCRF-CEM) upon TET1 KD. List of genes used in GSEA analysis in human T-ALL (CCRF-CEM) upon ectopic TET2 expression. Gene lists used for intersection analysis of MYC target genes, TET1 DEGs, and TET2 DEGs. 


\section{Acknowledgements}

We thank Dr. Dean W. Felsher, Stanford University, for kindly providing the mouse T-ALL cell lines. Furthermore, we thank Dr. Honglin Li, Augusta University, and the members of the van Riggelen laboratory for their support and useful suggestions.

\section{Authors' contributions}

CJP, AL, and JVR designed the experiments; CJP and AL performed the experiments and analyzed the data; CJP, AL, JHC, and JVR provided critical feedback; and CJP and JVR wrote the manuscript. All authors read and approved the final manuscript.

\section{Funding}

This work was supported by an American Cancer Society Institutional Research Grant \#IRG14-193-01 (J.v.R.).

\section{Availability of data and materials}

The data sets generated and/or analyzed during the current study are available in the GEO repository, under accession number GSE126029.

\section{Ethics approval and consent to participate}

Not applicable.

\section{Consent for publication}

Not applicable.

\section{Competing interests}

The authors declare that they have no competing interests.

\section{Author details}

1 Department of Biochemistry and Molecular Biology, Augusta University, 1410 Laney-Walker Blvd., Augusta, GA 30912, USA. ${ }^{2}$ Georgia Cancer Center, Augusta University, 1410 Laney-Walker Blvd., Augusta, GA 30912, USA.

Received: 6 March 2019 Accepted: 17 May 2019

Published online: 02 July 2019

\section{References}

1. Dang CV, O'Donnell KA, Zeller KI, Nguyen T, Osthus RC, Li F. The c-Myc target gene network. Semin Cancer Biol. 2006;16:253-64.

2. Grandori C, Cowley SM, James LP, Eisenman RN. The Myc/Max/Mad network and the transcriptional control of cell behavior. Annu Rev Cell Dev Biol. 2000;16:653-99.

3. Dang CV. c-Myc target genes involved in cell growth, apoptosis, and metabolism. Mol Cell Biol. 1999;19:1-11.

4. Eilers M, Eisenman RN. Myc's broad reach. Genes Dev. 2008;22:2755-66.

5. Felsher DW, Bishop JM. Reversible tumorigenesis by MYC in hematopoietic lineages. Mol Cell. 1999:4:199-207.

6. Shachaf CM, Kopelman AM, Arvanitis C, Karlsson A, Beer S, Mandl S, Bachmann MH, Borowsky AD, Ruebner B, Cardiff RD, et al. MYC inactivation uncovers pluripotent differentiation and tumour dormancy in hepatocellular cancer. Nature. 2004;431:1112-7.

7. Soucek L, Whitfield J, Martins CP, Finch AJ, Murphy DJ, Sodir NM, Karnezis AN, Swigart LB, Nasi S, Evan Gl. Modelling Myc inhibition as a cancer therapy. Nature. 2008:455:679-83.

8. Frank SR, Parisi T, Taubert S, Fernandez P, Fuchs M, Chan HM, Livingston DM, Amati B. MYC recruits the TIP60 histone acetyltransferase complex to chromatin. EMBO Rep. 2003;4:575-80.

9. McMahon SB, Wood MA, Cole MD. The essential cofactor TRRAP recruits the histone acetyltransferase hGCN5 to c-Myc. Mol Cell Biol. 2000;20:556-62.

10. Wolf E, Lin CY, Eilers M, Levens DL. Taming of the beast: shaping Mycdependent amplification. Trends Cell Biol. 2015;25:241-8.

11. Lin CY, Loven J, Rahl PB, Paranal RM, Burge CB, Bradner JE, Lee TI, Young RA. Transcriptional amplification in tumor cells with elevated c-Myc. Cell. 2012;151:56-67.

12. Nie Z, Hu G, Wei G, Cui K, Yamane A, Resch W, Wang R, Green DR, Tessarollo $\mathrm{L}$, Casellas $\mathrm{R}$, et al. c-Myc is a universal amplifier of expressed genes in lymphocytes and embryonic stem cells. Cell. 2012;151:68-79.
13. Sabo A, Kress TR, Pelizzola M de Pretis S, Gorski MM, Tesi A, Morelli MJ, Bora P, Doni M, Verrecchia A, et al. Selective transcriptional regulation by Myc in cellular growth control and lymphomagenesis. Nature. 2014;511:488-92.

14. Walz S, Lorenzin F, Morton J, Wiese KE, von Eyss B, Herold S, Rycak L, Dumay-Odelot H, Karim S, Bartkuhn M, et al. Activation and repression by oncogenic MYC shape tumour-specific gene expression profiles. Nature. 2014;511:483-7.

15. Knoepfler PS, Zhang XY, Cheng PF, Gafken PR, McMahon SB, Eisenman RN. Myc influences global chromatin structure. EMBO J. 2006;25:2723-34.

16. Wu CH, van Riggelen J, Yetil A, Fan AC, Bachireddy P, Felsher DW. Cellular senescence is an important mechanism of tumor regression upon c-Myc inactivation. Proc Natl Acad Sci USA. 2007;104:13028-33.

17. LiY, Choi PS, Casey SC, Dill DL, Felsher DW. MYC through miR-17-92 suppresses specific target genes to maintain survival, autonomous proliferation, and a neoplastic state. Cancer Cell. 2014;26:262-72.

18. Zhu P, Martin E, Mengwasser J, Schlag P, Janssen KP, Gottlicher M. Induction of HDAC2 expression upon loss of APC in colorectal tumorigenesis. Cancer Cell. 2004:5:455-63.

19. Poole CJ, van Riggelen J. MYC-Master Regulator of the Cancer Epigenome and Transcriptome. Genes (Basel). 2017;8:142.

20. Secombe J, Li L, Carlos L, Eisenman RN. The Trithorax group protein Lid is a trimethyl histone $\mathrm{H} 3 \mathrm{~K} 4$ demethylase required for dMyc-induced cell growth. Genes Dev. 2007;21:537-51.

21. van Riggelen J, Muller J, Otto T, Beuger V, Yetil A, Choi PS, Kosan C, Moroy $\mathrm{T}$, Felsher DW, Eilers M. The interaction between Myc and Miz1 is required to antagonize TGFbeta-dependent autocrine signaling during lymphoma formation and maintenance. Genes Dev. 2010;24:1281-94.

22. Brenner C, Fuks F. DNA methyltransferases: facts, clues, mysteries. Curr Top Microbiol Immunol. 2006;301:45-66

23. Kulis M, Esteller M. DNA methylation and cancer. Adv Genet. 2010;70:27-56

24. Jones PA, Baylin SB. The fundamental role of epigenetic events in cancer. Nat Rev Genet. 2002;3:415-28.

25. Shen H, Laird PW. Interplay between the cancer genome and epigenome. Cell. 2013;153:38-55.

26. Poole CJ, Zheng W, Lodh A, Yevtodiyenko A, Liefwalker D, Li H, Felsher DW, van Riggelen J. DNMT3B overexpression contributes to aberrant DNA methylation and MYC-driven tumor maintenance in T-ALL and Burkitt's lymphoma. Oncotarget. 2017;8:76898-920.

27. Bhattacharyya S, Yu Y, Suzuki M, Campbell N, Mazdo J, Vasanthakumar A, Bhagat TD, Nischal S, Christopeit M, Parekh S, et al. Genome-wide hydroxymethylation tested using the HELP-GT assay shows redistribution in cancer. Nucleic Acids Res. 2013;41:e157.

28. Ficz G, Branco MR, Seisenberger S, Santos F, Krueger F, Hore TA, Marques CJ, Andrews S, Reik W. Dynamic regulation of 5-hydroxymethylcytosine in mouse ES cells and during differentiation. Nature. 2011;473:398-402.

29. Mellen M, Ayata P, Dewell S, Kriaucionis S, Heintz N. MeCP2 binds to $5 \mathrm{hmC}$ enriched within active genes and accessible chromatin in the nervous system. Cell. 2012;151:1417-30.

30. Song CX, Szulwach KE, Fu Y, Dai Q, Yi C, Li X, Li Y, Chen CH, Zhang W, Jian $X$, et al. Selective chemical labeling reveals the genome-wide distribution of 5-hydroxymethylcytosine. Nat Biotechnol. 2011;29:68-72.

31. Mitrea C, Wijesinghe P, Dyson G, Kruger A, Ruden DM, Draghici S, BolligFischer A. Integrating $5 \mathrm{hmC}$ and gene expression data to infer regulatory mechanisms. Bioinformatics. 2018;34:1441-7.

32. Yetil A, Anchang B, Gouw AM, Adam SJ, Zabuawala T, Parameswaran R, van Riggelen J, Plevritis S, Felsher DW. p19ARF is a critical mediator of both cellular senescence and an innate immune response associated with MYC inactivation in mouse model of acute leukemia. Oncotarget. 2015;6:3563-77.

33. Haferlach T, Kohlmann A, Wieczorek L, Basso G, Kronnie GT, Bene MC, De Vos J, Hernandez JM, Hofmann WK, Mills Kl, et al. Clinical utility of microarray-based gene expression profiling in the diagnosis and subclassification of leukemia: report from the International Microarray Innovations in Leukemia Study Group. J Clin Oncol. 2010;28:2529-37.

34. Wu CH, Sahoo D, Arvanitis C, Bradon N, Dill DL, Felsher DW. Combined analysis of murine and human microarrays and ChIP analysis reveals genes associated with the ability of MYC to maintain tumorigenesis. PLoS Genet. 2008:4:e1000090. 
35. Zeller KI, Jegga AG, Aronow BJ, O'Donnell KA, Dang CV. An integrated database of genes responsive to the Myc oncogenic transcription factor: identification of direct genomic targets. Genome Biol. 2003;4:R69.

36. Subramanian A, Tamayo P, Mootha VK, Mukherjee S, Ebert BL, Gillette MA, Paulovich A, Pomeroy SL, Golub TR, Lander ES, Mesirov JP. Gene set enrichment analysis: a knowledge-based approach for interpreting genome-wide expression profiles. Proc Natl Acad Sci USA 2005;102:15545-50.

37. Liberzon A, Subramanian A, Pinchback R, Thorvaldsdottir $H$, Tamayo $P$, Mesirov JP. Molecular signatures database (MSigDB) 3.0. Bioinformatics. 2011;27:1739-40.

38. Rasmussen KD, Helin K. Role of TET enzymes in DNA methylation, development, and cancer. Genes Dev. 2016;30:733-50.

39. Ko M, An J, Pastor WA, Koralov SB, Rajewsky K, Rao A. TET proteins and 5-methylcytosine oxidation in hematological cancers. Immunol Rev. 2015;263:6-21.

40. Zhang $W, X u$ J. DNA methyltransferases and their roles in tumorigenesis. Biomark Res. 2017;5:1

41. Wang J, Li F, Ma Z, Yu M, Guo Q, Huang J, Yu W, Wang Y, Jin J. High expression of TET1 predicts poor survival in cytogenetically normal acute myeloid leukemia from two cohorts. EBioMedicine. 2018;28:90-6.

42. Huang $H$, Jiang $X$, Wang J, Li Y, Song CX, Chen P, Li S, Gurbuxani S, Arnovitz S, Wang Y, et al. Identification of MLL-fusion/MYC dash, verticalmiR-26 dash, verticalTET1 signaling circuit in MLL-rearranged leukemia. Cancer Lett. 2016;372:157-65.

43. Ittel A, Jeandidier E, Helias C, Perrusson N, Humbrecht C, Lioure B, Mazurier I, Mayeur-Rousse C, Lavaux A, Thiebault S, et al. First description of the $\mathrm{t}(10 ; 11)(\mathrm{q} 22 ; \mathrm{q} 23) / \mathrm{MLL}-\mathrm{TET} 1$ translocation in a T-cell lymphoblastic lymphoma, with subsequent lineage switch to acute myelomonocytic myeloid leukemia. Haematologica. 2013;98:e166-8.

44. Huang H, Jiang X, Li Z, Li Y, Song CX, He C, Sun M, Chen P, Gurbuxani S, Wang J, et al. TET1 plays an essential oncogenic role in MLL-rearranged leukemia. Proc Natl Acad Sci USA. 2013;110:11994-9.

45. Good CR, Panjarian S, Kelly AD, Madzo J, Patel B, Jelinek J, Issa JJ. TET1mediated hypomethylation activates oncogenic signaling in triple-negative breast cancer. Cancer Res. 2018;78:4126-37.

46. Frycz BA, Murawa D, Borejsza-Wysocki M, Marciniak R, Murawa P, Drews M, Kolodziejczak A, Tomela K, Jagodzinski PP. Decreased expression of ten-eleven translocation 1 protein is associated with some clinicopathological features in gastric cancer. Biomed Pharmacother. 2014;68:209-12.

47. Li Y, Shen Z, Jiang H, Lai Z, Wang Z, Jiang K, Ye Y, Wang S. MicroRNA4284 promotes gastric cancer tumorigenicity by targeting ten-eleven translocation 1. Mol Med Rep. 2018;17:6569-75.

48. Neri F, Dettori D, Incarnato D, Krepelova A, Rapelli S, Maldotti M, Parlato C, Paliogiannis P, Oliviero S. TET1 is a tumour suppressor that inhibits colon cancer growth by derepressing inhibitors of the WNT pathway. Oncogene. 2015;34:4168-76.

49. Yang L, Yu SJ, Hong Q, Yang Y, Shao ZM. Reduced expression of TET1, TET2, TET3 and TDG mRNAs are associated with poor prognosis of patients with early breast cancer. PLoS ONE. 2015;10:e0133896.
50. Collignon E, Canale A, AI Wardi C, Bizet M, Calonne E, Dedeurwaerder S, Garaud S, Naveaux C, Barham W, Wilson A, et al. Immunity drives TET1 regulation in cancer through NF-kappaB. Sci Adv. 2018;4:eaap7309.

51. Pei YF, Lei Y, Liu XQ. MiR-29a promotes cell proliferation and EMT in breast cancer by targeting ten eleven translocation 1. Biochim Biophys Acta. 2016;1862:2177-85.

52. van Riggelen J, Yetil A, Felsher DW. MYC as a regulator of ribosome biogenesis and protein synthesis. Nat Rev Cancer. 2010;10:301-9.

53. Mouly E, Ghamlouch H, Della-Valle V, Scourzic L, Quivoron C, Roos-Weil D, Pawlikowska P, Saada V, Diop MK, Lopez CK, et al. B-cell tumor development in Tet2-deficient mice. Blood Adv. 2018;2:703-14.

54. Ghaleb AM, Yang VW. Kruppel-like factor 4 (KLF4): what we currently know. Gene. 2017;611:27-37.

55. Cimmino L, Dolgalev I, Wang Y, Yoshimi A, Martin GH, Wang J, Ng $V$, Xia B, Witkowski MT, Mitchell-Flack M, et al. Restoration of TET2 function blocks aberrant self-renewal and leukemia progression. Cell. 2017;170(1079-1095):e1020.

56. Williams K, Christensen J, Pedersen MT, Johansen JV, Cloos PA, Rappsilber J, Helin K. TET1 and hydroxymethylcytosine in transcription and DNA methylation fidelity. Nature. 2011;473:343-8.

57. Wutz A, Smrzka OW, Schweifer N, Schellander K, Wagner EF, Barlow DP. Imprinted expression of the Igf2r gene depends on an intronic CpG island. Nature. 1997;389:745-9.

58. Zhang X, Wu M, Xiao H, Lee MT, Levin L, Leung YK, Ho SM. Methylation of a single intronic CpG mediates expression silencing of the PMP24 gene in prostate cancer. Prostate. 2010;70:765-76.

59. Pajic A, Spitkovsky D, Christoph B, Kempkes B, Schuhmacher M, Staege MS, Brielmeier M, Ellwart J, Kohlhuber F, Bornkamm GW, et al. Cell cycle activation by c-myc in a burkitt lymphoma model cell line. Int J Cancer. 2000;87:787-93.

60. Kim D, Langmead B, Salzberg SL. HISAT: a fast spliced aligner with low memory requirements. Nat Methods. 2015;12:357-60.

61. Pertea M, Pertea GM, Antonescu CM, Chang TC, Mendell JT, Salzberg SL. StringTie enables improved reconstruction of a transcriptome from RNAseq reads. Nat Biotechnol. 2015;33:290-5.

62. Frazee AC, Pertea G, Jaffe AE, Langmead B, Salzberg SL, Leek JT. Ballgown bridges the gap between transcriptome assembly and expression analysis. Nat Biotechnol. 2015;33:243-6.

63. Shen L, Shao NY, Liu X, Maze I, Feng J, Nestler EJ. diffReps: detecting differential chromatin modification sites from ChIP-seq data with biological replicates. PLoS ONE. 2013;8:e65598.

64. da Huang W, Sherman BT, Lempicki RA. Systematic and integrative analysis of large gene lists using DAVID bioinformatics resources. Nat Protoc. 2009:4:44-57.

\section{Publisher's Note}

Springer Nature remains neutral with regard to jurisdictional claims in published maps and institutional affiliations.

Ready to submit your research? Choose BMC and benefit from

- fast, convenient online submission

- thorough peer review by experienced researchers in your field

- rapid publication on acceptance

- support for research data, including large and complex data types

- gold Open Access which fosters wider collaboration and increased citations

- maximum visibility for your research: over 100M website views per year

At BMC, research is always in progress.

Learn more biomedcentral.com/submissions 